\title{
Analysis of the effect of intermittency in a high-pressure turbine blade
}

\author{
Dorian Dupuy*1, Laurent Gicquel ${ }^{1}$, Nicolas Odier ${ }^{1}$, Florent Duchaine $^{1}$, and Tony Arts ${ }^{2}$ \\ ${ }^{1}$ European Centre for Research and Advanced Training in Scientific Computing, Toulouse F-31057 Cedex \\ 1, France \\ ${ }^{2}$ von Karman Institute for Fluid Dynamics, Rhode-Saint-Genèse B-1640, Belgium \\ (Published version: Physics of Fluids 32(9), 095101 (2021); https://doi.org/10.1063/5.0018679)
}

\begin{abstract}
High-pressure turbine blades are subject to large thermomechanical loads that may threaten their mechanical integrity. The prediction of the heat transfer on the blade surface, crucial to ensure its durability, thus requires an accurate description of the flow physics around the blade to be reliable. In an effort to better qualify the use of computational fluid dynamics in this design context as well as the need for an improved understanding of the flow physics, this paper investigates a transonic highly loaded linear turbine blade cascade that has been found difficult to predict in the literature using large-eddy simulations. Indeed, the configuration results in shocks and acoustic waves on the suction side of the blade, features that are commonly encountered in high-pressure turbines. Turbulent spots are observed on the suction-side boundary layer with an inlet turbulence intensity of $6 \%$. The turbulent spots are shown to have a complex and highly unsteady effect on the shock/boundary-layer interaction, disrupting flow detachment and creating laminar spots downstream of the shock. To address these transient flow phenomena, conditional averages based on the intermittency level are introduced to show that accurate heat transfer predictions require an accurate prediction of the rate of turbulent-spot production. The analysis then focuses on the effect of intermittency on the turbulent kinetic energy exchanges in the near-wall region, as the turbulent kinetic energy balance must be addressed in Reynolds-averaged Navier-Stokes models.
\end{abstract}

\section{Introduction}

It is well known that gas turbines must operate at high temperature to maximise efficiency. However, this imposes large thermal and thermomechanical loads on their components. The nozzle guide vane of high-pressure turbines, located directly downstream of the combustion chamber, is particularly vulnerable. An accurate prediction of the heat transfer on the nozzle blade surface is thus crucial to ensure the mechanical integrity of the blade and the engine. The aerothermal behaviour of a blade is determined by the complex flow physics of high-pressure turbines, which may encounter large-scale inflow perturbations and accumulate shocks, transition, separation bubbles, relaminarisation at the leading edge and vortex shedding at the trailing edge. High-pressure turbines are challenging for numerical or experimental investigations because of the large Reynolds number and transonic exit Mach number, the high-frequency unsteadiness, the large number of scales involved and the large temperatures, hindering direct measurements and optical access [60, 36]. In this context, the experimental campaign of Arts et al. [1] provides a valuable database of wall heat flux distribution for an uncooled high-pressure turbine nozzle with a simplified twodimensional geometry and lower temperature levels. A range of inlet turbulence intensity, Reynolds and Mach numbers is reported, demonstrating the importance of the inlet turbulence level on wall heat transfer.

Due to the above-discussed reasons, the numerical investigation of the linear blade cascade of Arts et al. [1], hereafter called VKI-LS89, has been addressed by a number of authors [29, 60]. Direct numerical simulations (DNS), in which all scales of turbulence are assumed to be resolved, were carried out by Wheeler et al. [75]. This was then used by Zauner et al. [79] to predict,

*Corresponding author : dorian.dupuy@cerfacs.fr 
from stability analysis, the predominant frequencies close to the trailing edge, while Sandberg and Wheeler [61] investigated the occurrence and sensitivity of acoustic feedback loops. In parallel, Hoarau et al. [26] investigated the effect of a dense gas equation of state on the development of shock systems and turbulence transition. Lin et al. [38] studied the development of the wake vortices and analysed the local entropy generation rate [37]. Zhao and Sandberg [80] characterised the effects of freestream turbulence on the generation of vortical structures at the leading edge and on transition mechanisms. Conventional Reynolds-averaged Navier-Stokes (RANS) models have been found unable to accurately predict the wall heat transfer for high inlet turbulence intensity $[7,19,39,17]$. The prediction of the location and length of the turbulent transition is in this respect crucial, since it is associated with a rapid increase in heat flux $[52,53]$. Large-eddy simulations (LES) are a possible alternative based on the explicit resolution of large scales and the modelling of the interactions between small and large scales. Large-eddy simulations of the VKI-LS89 blade cascade include Bhaskaran and Lele [3], Fransen et al. [17], Gourdain et al. [21], Collado Morata et al. [10], Pichler et al. $[49,50]$ and Segui et al. [67, 68]. Several studies [10, 67] pointed out the influence of the characteristics of the inflow turbulence and its decay on the wall heat transfer. In particular, uncertainties concerning the turbulence integral length scale, not provided in the original experiments [1], may affect turbulence transition and thus the aerothermal behaviour of the blade $[50,80]$. Although large-eddy simulations offer a greater fidelity than RANS simulations, the accuracy of the predictions varies depending on the exact operating conditions. In particular, an agreement with the experimental data of Arts et al. [1] is obtained for the operating conditions of the MUR129 test case [10, 21, 67] while the wall heat flux on the suction side of the blade surface is consistently underpredicted for the MUR235 test case, which is relatively similar but has a larger inlet turbulence intensity of $6 \%$. This suggests that these flow conditions are associated with particularly complex flow features and boundary layer dynamics, which remain difficult to capture numerically.

In this paper, highly resolved large-eddy simulations of the VKI-LS89 blade cascade are carried out for two sets of operating conditions corresponding to the MUR129 and MUR235 test cases of Arts et al. [1]. The simulations are performed on a hybdrid unstructured mesh using a finiteelement method in a cell-vertex formulation and a two-step Taylor-Galerkin scheme. A synthetic inflow turbulence injection is used at the inlet. It is shown that the wall heat transfer is in the MUR235 test case influenced by the occurrence of turbulent spots in a transitional region upstream of the shock, whereas no turbulent spots are identified in the numerical simulation of the MUR129 test case [1] which has a low inlet turbulence intensity of $0.8 \%$. The dynamics of the turbulent spots is analysed by comparing their growth rate to the zero-pressure-gradient (ZPG) incompressible flat-plate case, confirming their physical relevance. The interaction of the turbulent spots with the shocks as well as the transient separation of the flow is also examined. It is noted from these analyses that an accurate prediction of the wall heat flux requires an accurate prediction of the intermittency level on the suction side of the blade and thus of the dynamics and production rate of the turbulent spots, currently underestimated in available large-eddy simulations of the MUR235 test case. The influence of the turbulent spots on viscous dissipation and on the production of turbulent kinetic energy in the transitional region and around the shocks is finally addressed, as the kinetic energy balance is critical for the prediction of losses and the modelling of the RANS equations.

The paper is organised as follows. The flow configuration and the numerical procedure are presented in section 2. The dynamics of the turbulent spots is analysed in section 3 . The effect of the turbulent spots on boundary layer statistics is studied in section 4 .

\section{Flow Configuration and computational setup}

\subsection{Flow configuration}

The high-pressure linear nozzle blade cascade of Arts et al. [1] is considered. The blade geometry is an extruded two-dimensional profile, without spanwise curvature. The chord $c$ is 67.647 $\mathrm{mm}$. The simulations address two sets of operating conditions corresponding to the MUR235 and MUR129 test cases of Arts et al. [1] and reported in Table 1. In both cases, the flow is compressible 


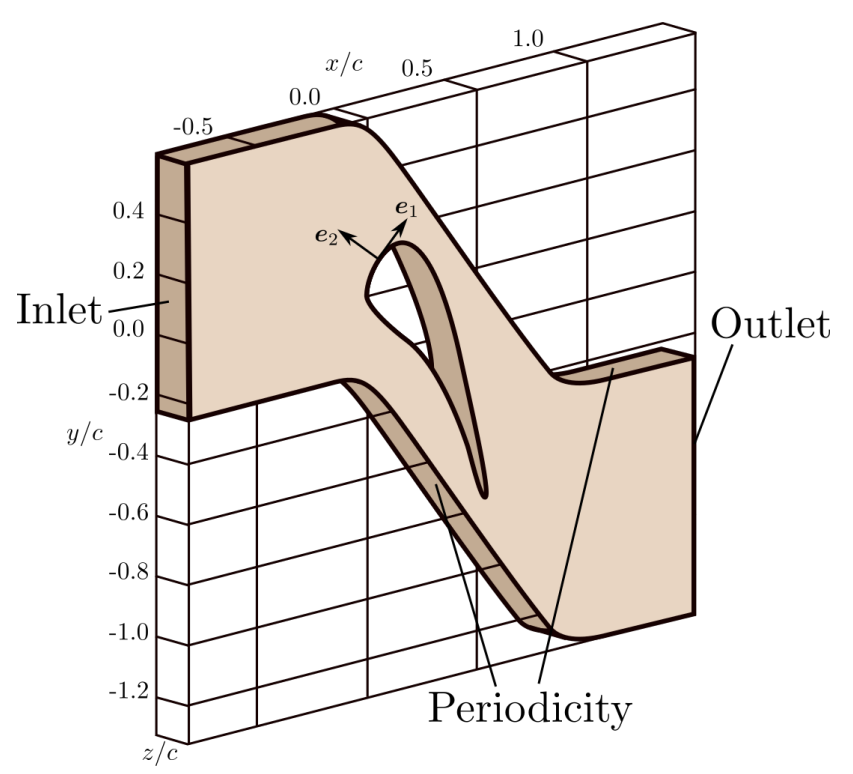

Figure 1: Computational domain of the simulation.

and transonic. The Mach number, the Reynolds number and the temperature ratio between the inlet and the blade surface are representative of typical high-pressure turbines. The MUR235 and MUR129 test cases have very similar operating conditions except for the inlet turbulence intensity which is low in the MUR129 test case $\left(T u_{\text {inlet }}=0.8 \%\right)$ and large in the MUR235 test case $\left(T u_{\text {inlet }}\right.$ $=6 \%)$. For the purpose of the simulation, the manufacturing coordinates of the blade profile have been reinterpolated following Wheeler et al. [75] to ensure a smooth surface curvature. The experimental setup is modelled using a biperiodic computational domain, represented in Figure 1, as justified in the original report [1]. The pitchwise periodicity reproduces an infinite linear blade cascade. The spanwise periodicity assumes statistical homogeneity in this direction. For latter use, two coordinate systems are defined: a Cartesian coordinate system $(x, y, z)$ associated with the standard basis $\left\{\boldsymbol{e}_{\boldsymbol{x}}, \boldsymbol{e}_{\boldsymbol{y}}, \boldsymbol{e}_{\boldsymbol{z}}\right\}$, with $\boldsymbol{e}_{\boldsymbol{x}}$ the axial direction, $\boldsymbol{e}_{\boldsymbol{y}}$ the pitchwise direction and $\boldsymbol{e}_{\boldsymbol{z}}$ the spanwise direction; and a curvilinear coordinate system $\left(s_{1}, s_{2}, z\right)$ associated with the basis $\left\{\boldsymbol{e}_{\mathbf{1}}, \boldsymbol{e}_{\mathbf{2}}, \boldsymbol{e}_{\boldsymbol{z}}\right\}$, where $\boldsymbol{e}_{1}$ and $\boldsymbol{e}_{\mathbf{2}}$ are unit vectors tangent and normal to the blade surface respectively. The origin of the two coordinate systems is placed at the leading edge of the blade. At this point, the curvilinear unit vector $\boldsymbol{e}_{\mathbf{1}}$ is oriented positively towards the suction side of the blade. The inlet axial plane is located at $x_{\text {inlet }} / c=-0.81$ and the outlet axial plane at $x_{\text {outlet }} / c=1.48$. The spanwise length of the domain is $L_{z} / c=0.148$. The sensitivity analysis of Collado Morata et al. [10] suggests that this value is sufficient to prevent the flow structures from being constrained by the spanwise extent of the computational domain. Besides, this spanwise range is within the range of the values found in the literature $[3,17,49]$.

The flow is modelled by a continuous medium in local thermodynamic equilibrium and following the compressible Navier-Stokes equations without any body force or heat source. The fluid (air) is assumed to obey the ideal gas equation of state, which is a reasonable assumption since the operating conditions are near ambient pressure and ambient temperature. The viscous shear stresses are computed assuming a Newtonian fluid under Stokes' hypothesis. Fourier's law is used to compute the conductive heat flux. Note that no combustion process is occurring in the experiment. Using a large-eddy simulation formalism in the computable filtered total energy formulation, the governing equations may be written using Einstein summation convention as:

$$
\begin{gathered}
\frac{\partial \rho}{\partial t}+\frac{\partial \rho U_{j}}{\partial x_{j}}=0 \\
\frac{\partial \rho U_{i}}{\partial t}+\frac{\partial \rho U_{j} U_{i}}{\partial x_{j}}=-\frac{\partial P}{\partial x_{i}}+\frac{\partial \Sigma_{i j}(\boldsymbol{U}, T)}{\partial x_{j}}, \\
\frac{\partial \rho E}{\partial t}+\frac{\partial \rho U_{j} H}{\partial x_{j}}=-\frac{\partial Q_{j}(T)}{\partial x_{j}}-\frac{\partial U_{j} P}{\partial x_{j}}+\frac{\partial U_{j} \Sigma_{i j}(\boldsymbol{U}, T)}{\partial x_{j}},
\end{gathered}
$$




\begin{tabular}{lll} 
& MUR235 & MUR129 \\
\hline Inlet stagnation pressure $P_{s, \text { inlet }}, \mathrm{Pa}$ & $1.828 \times 10^{5}$ & $1.849 \times 10^{5}$ \\
Inlet stagnation temperature $T_{s, \text { inlet }}, \mathrm{K}$ & 413.3 & 409.2 \\
Inlet Reynolds number $R e_{\text {inlet }}$ & $2.647 \times 10^{5}$ & $2.710 \times 10^{5}$ \\
Inlet Mach number $M a_{\text {inlet }}$ & 0.150 & 0.150 \\
Inlet turbulence intensity $T u_{\text {inlet }}$ & $6 \%$ & $0.8 \%$ \\
Outlet pressure $P_{\text {outlet }}, \mathrm{Pa}$ & $1.049 \times 10^{5}$ & $1.165 \times 10^{5}$ \\
Outlet Reynolds number $R e_{\text {outlet }}$ & $1.152 \times 10^{6}$ & $1.135 \times 10^{6}$ \\
Outlet Mach number $M a_{\text {outlet }}$ & 0.927 & 0.840 \\
Wall temperature $T_{w}, \mathrm{~K}$ & 301.2 & 297.8
\end{tabular}

Table 1: Operating point conditions of the MUR235 and MUR129 test cases [1].

with $\rho$ the filtered density, $t$ the time, $\boldsymbol{U}$ the Favre-filtered velocity, $\boldsymbol{x}$ the Cartesian coordinate, $P$ the filtered pressure, $T$ the Favre-filtered temperature, $E$ the Favre-filtered total energy per unit mass and $H=E+P / \rho$ the Favre-filtered total enthalpy per unit mass. The tabulated data of Stull and Prophet [71] are used to compute temperature from internal energy $e$, defined as $e=E-\frac{1}{2} U_{i} U_{i}$. The viscous shear stress and conductive heat flux include the modelled subgrid-scale contribution,

$$
\begin{gathered}
\Sigma_{i j}(\boldsymbol{U}, T)=\left(\mu(T)+\mu_{\mathrm{sgs}}\right)\left(\frac{\partial U_{i}}{\partial x_{j}}+\frac{\partial U_{j}}{\partial x_{i}}-\frac{2}{3} \frac{\partial U_{k}}{\partial x_{k}} \delta_{i j}\right), \\
Q_{j}(T)=-\left(\lambda(T)+\lambda_{\mathrm{sgs}}\right) \frac{\partial T}{\partial x_{j}}
\end{gathered}
$$

where $\delta_{i j}$ denotes the Kronecker delta. Sutherland's law is used to compute the dynamic viscosity [72], $\mu(T)=\mu_{0}\left(T / T_{0}\right)^{3 / 2}\left(T_{0}+T_{1}\right) /\left(T+T_{1}\right)$, with $\mu_{0}=1.716 \times 10^{-5} \mathrm{~Pa} \mathrm{~s}, T_{0}=273.15 \mathrm{~K}$ and $T_{1}=110.6 \mathrm{~K}$, and a constant Prandtl number $\operatorname{Pr}=0.71$ is assumed to compute thermal conductivity, $\lambda(T)=C_{p} \mu(T) / P r$. The WALE model is used to compute the subgrid-scale viscosity [44], with a model constant $C_{w}=0.57$, and a constant subgrid-scale Prandtl number $\operatorname{Pr}_{\text {sgs }}=0.6$ is assumed to compute subgrid-scale thermal conductivity, $\lambda_{\mathrm{sgs}}=C_{p} \mu_{\mathrm{sgs}} / P r_{\mathrm{sgs}}$.

\subsection{Numerical setup}

The numerical study is conducted using a finite-element method in a cell-vertex formulation. A two-step Taylor-Galerkin scheme with third-order spatial and temporal accuracy [9] is used for convection and a centred second-order scheme is used for diffusion. The boundary conditions match the operating conditions of the experiment. At the inlet, stagnation pressure and stagnation temperature are imposed using a non-reflecting boundary condition based on characteristic analysis [46], and turbulence is injected using the approach of Kraichnan [32] with an integral length scale of $3.19 \mathrm{~mm}$. The study of Segui et al. [67] suggests that synthetic turbulence injection is a reasonable choice in the present configuration due to the distance between the inlet plane and the leading edge of the blade. At the outlet, pressure is imposed using the non-reflecting boundary condition of Granet et al. [22]. The blade wall surface has an imposed uniform temperature and uses a no-slip condition. The numerical simulation is performed using the hybrid flow solver AVBP [65].

For the MUR129 test case, the mesh contains $213.0 \times 10^{6}$ hybrid cells: the near-wall region is discretised by 15 layers of triangular prism, while the rest of the domain is discretised with tetrahedra. The total number of prisms is $89.5 \times 10^{6}$ and the total number of nodes $135.4 \times 10^{6}$. A finer mesh is used for the MUR235 test case: the mesh contains $586.8 \times 10^{6}$ hybrid cells with 20 prismatic layers in the near-wall region, amounting to $269.4 \times 10^{6}$ prisms in total and $386.2 \times 10^{6}$ nodes. The numerical setup is summarised in Table 2. A study of the effect of mesh refinement on the predictions is provided in appendix A. For post-processing purpose, a constant timestep is used. The timestep is $\Delta t / t_{c}=3.9 \times 10^{-6}$, where $t_{c}=c / U_{\text {inlet }}$ is the inlet flow characteristic time based on the chord of the blade $c$. This corresponds to a CFL number of 0.9. Averaged flow statistics are computed assuming ergodicity as averages over time and over the spanwise direction. The averaging duration is 1.3 inlet flow characteristic times. 


\begin{tabular}{lcc} 
& MUR235 & MUR129 \\
\hline Total number of cells & $586.8 \times 10^{6}$ & $213.6 \times 10^{6}$ \\
Total number of prisms & $269.4 \times 10^{6}$ & $89.5 \times 10^{6}$ \\
Total number of nodes & $386.2 \times 10^{6}$ & $135.4 \times 10^{6}$ \\
Number of prismatic layers & 20 & 15
\end{tabular}

Table 2: Numerical parameters of the simulations.

\subsection{Validation}

The instantaneous visualisations given in Figure 2 compares the flow fields obtained for the MUR235 and MUR129 test cases. In the MUR235 test case, there is a series of shocks quasinormal to the blade suction side. The shocks are in interaction with the suction-side boundary layer as well as the wake vortex shedding generated at the trailing edge of the blade. A series of acoustic waves, also generated at the trailing edge, is impinging on the suction side and reflected by the blade surface. In the MUR129 test case, similar acoustic waves patterns are generated but no shock is visible. The mean heat transfer coefficient obtained in the MUR235 and MUR129 test cases is compared to the experimental data of Arts et al. [1] in Figure 3. The heat transfer coefficient is in the following defined as

$$
h=\frac{\left\langle Q_{w}\right\rangle}{T_{s, \text { inlet }}-\left\langle T_{w}\right\rangle},
$$

where $Q_{w}$ is the wall heat flux and $T_{w}$ the wall temperature. There is in both cases an overall agreement between the simulations and the experiment for the shape of the heat transfer distribution. In the MUR129 test case, the wall heat transfer is well predicted throughout the blade. The wall heat transfer is also well predicted in the MUR235 on the pressure side although slightly underestimated by the simulation. On the suction side, the heat transfer level is in agreement with the experiment downstream of the shocks and in the strongly accelerating region near the leading edge. However, the simulation predicts based on these time-averaged results a heat transfer decrease from $s_{1} / c=0.5$ to 0.9 which is not present in the experimental data. The discrepancy nonetheless coincides, as detailed hereafter, with the presence in the simulation of a transitional region with turbulent spots (localised regions of turbulent flow [15]). Indeed, the boundary layer on the suction side of the blade is as shown in Figure 4 laminar near the leading edge, turbulent downstream of the shock and transitional from $s_{1} / c=0.5$ to 0.9 . The rest of this paper studies specifically the impact of the turbulent spots as observed in the MUR235 test case. The objective is to evidence their influence on the heat transfer between the fluid and the blade.

\subsection{Spot identification procedure}

In order to track the turbulent spots on the suction side of the blade, the identification procedure of Rehill et al. [57] is followed. The identification is performed on two-dimensional data, obtained from the interpolation of the three-dimensional flow fields on an offset surface P1 at a fixed distance from the suction-side blade wall. The distance of the offset surface from the wall is given in Figure 5 with the wall scaling $s_{2}^{+}=s_{2} U_{\tau} / \nu_{\omega}$, where $\omega$ denotes wall values, $U_{\tau}=\sqrt{\nu_{\omega}\left(\partial_{2}\left\langle U_{1}\right\rangle\right)_{\omega}}$ is the friction velocity and $\langle\cdot\rangle$ denotes the statistical average operator. This offset surface contains 100 cells in the spanwise direction and 678 cells following the curvilinear abscissa. The identification procedure involves three main steps:

1. First, the instantaneous field of $Q$ criterion is interpolated on the offset surface P1. Identification procedures based on the $Q$ criterion are less sensitive than methods based on the instantaneous velocity components, the $\lambda_{2}$ criterion or the gradient of the finite-time Lyapunov exponent and are effective in the presence of organised streaks in a zero-pressure-gradient transitional boundary layer [57]. In order to disregard variations of the $Q$ criterion not related to turbulence, the fluctuation of the $Q$ criterion with respect to the median is used,

$$
\grave{Q}\left(s_{1}, z\right)=Q\left(s_{1}, z\right)-\operatorname{median}(Q)\left(s_{1}\right)
$$



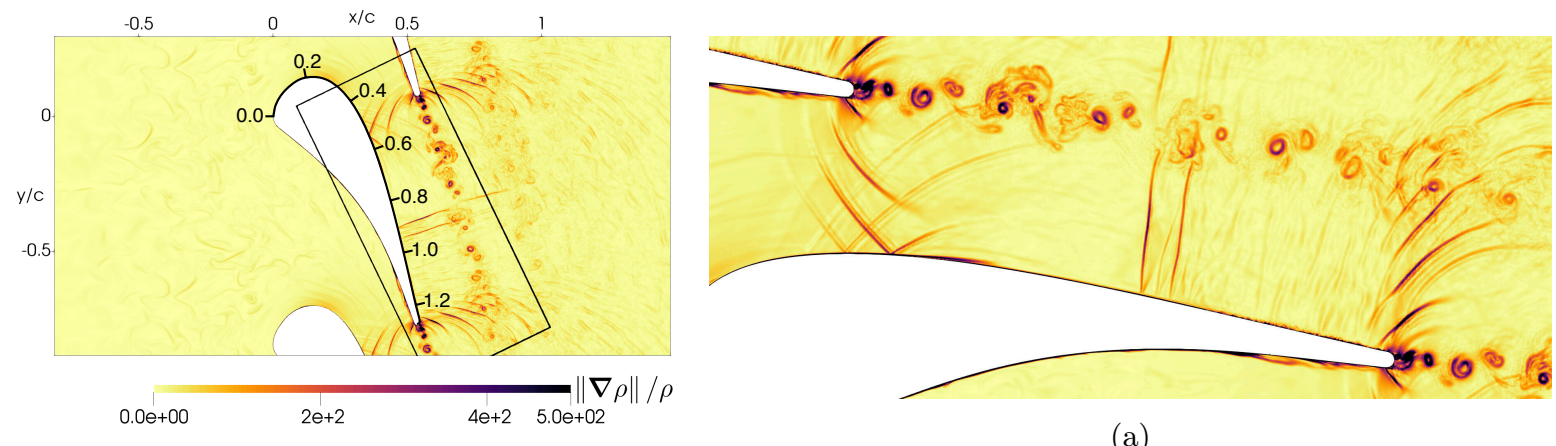

(a)
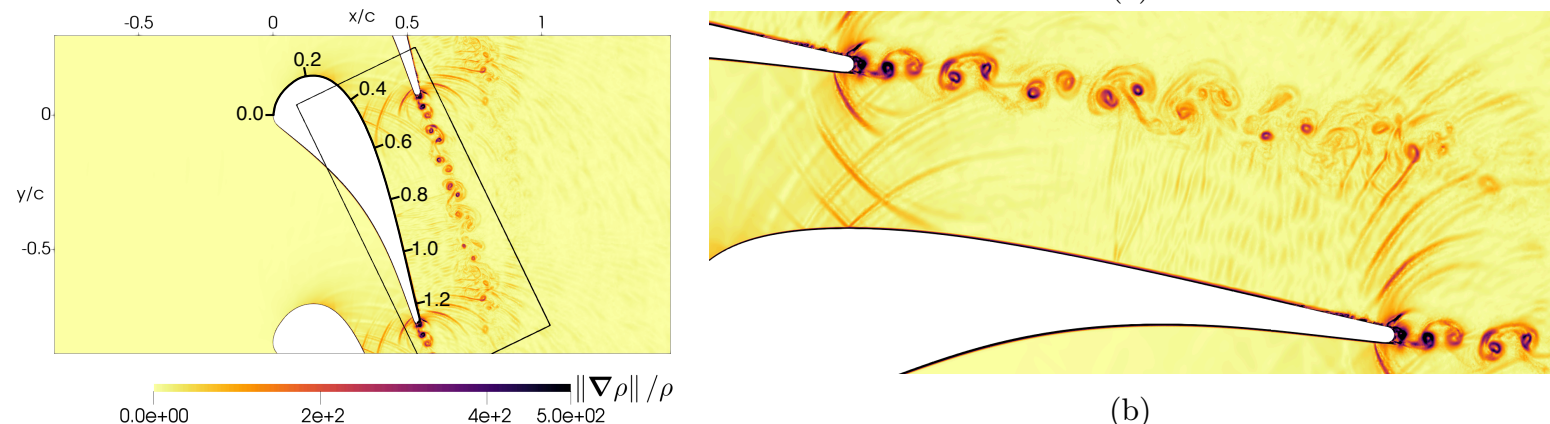

(b)

Figure 2: Instantaneous field of $\|\nabla \rho\| / \rho$ on the plane $z / c=0.037$ in the test case MUR235 (a) and MUR129 (b). The curvilinear abscissa $s_{1} / c$ along the blade is given in the left figure for reference.

with $Q=(1 / 2)\left(\|\boldsymbol{\Omega}\|^{2}-\left\|\mathbf{S}^{\mathbf{d}}\right\|^{2}\right)$ and where $\|\cdot\|$ is the Euclidian matrix norm, $\boldsymbol{\Omega}=(1 / 2)(\nabla \boldsymbol{U}-$ $\left.(\nabla \boldsymbol{U})^{T}\right)$ the rate of rotation tensor and $\mathbf{S}^{\mathbf{d}}=(1 / 2)\left(\nabla \boldsymbol{U}+(\nabla \boldsymbol{U})^{T}\right)-(1 / 3) \mathbf{I}_{\mathbf{d}} \nabla \cdot \boldsymbol{U}$ the deviatoric rate of deformation tensor.

2. Second, a threshold is applied to the selected instantaneous field. The thresholded field may be expressed as

$$
T_{Q}\left(s_{1}, z\right)=\left[\left\|\grave{Q}\left(s_{1}, z\right)\right\|>\text { threshold }\right],
$$

where $[\cdot]$ denotes the Iverson bracket operator, evaluated to 1 if the logical proposition within brackets is satisfied and to 0 otherwise. In the following, the reference threshold is set to $0.5 \%$ of the maximum value.

3. Third, the thresholded fields are filtered and smoothed, removing spurious turbulent areas and holes within the turbulent regions. The filter switches a cell turbulent if there are more turbulent cells than laminar ones in the vicinity, and switches a cell laminar if it is surrounded by laminar cells. The resulting field contains a set of contiguous areas identified as turbulent, each of which is considered as a different spot.

The procedure is represented in Figure 6. By virtue of its two-dimensional nature, this identification method does not capture the dependency of the spot to the wall-normal coordinate and the spot motions away from the surface. Since turbulent spots typically have a constant growth rate very near the wall [76], the method can nonetheless be expected to be robust to small variations in the distance of the offset surface from the wall.

\section{Dynamics of the turbulent spots}

Instantaneous flow visualisations of the MUR235 test case on the offset surface P1 along the suction side of the blade are shown in Figure 7. Three main areas can be identified: a laminar region on the strongly accelerating region near the leading edge with elongated streaks $\left(s_{1} / c<\right.$ 


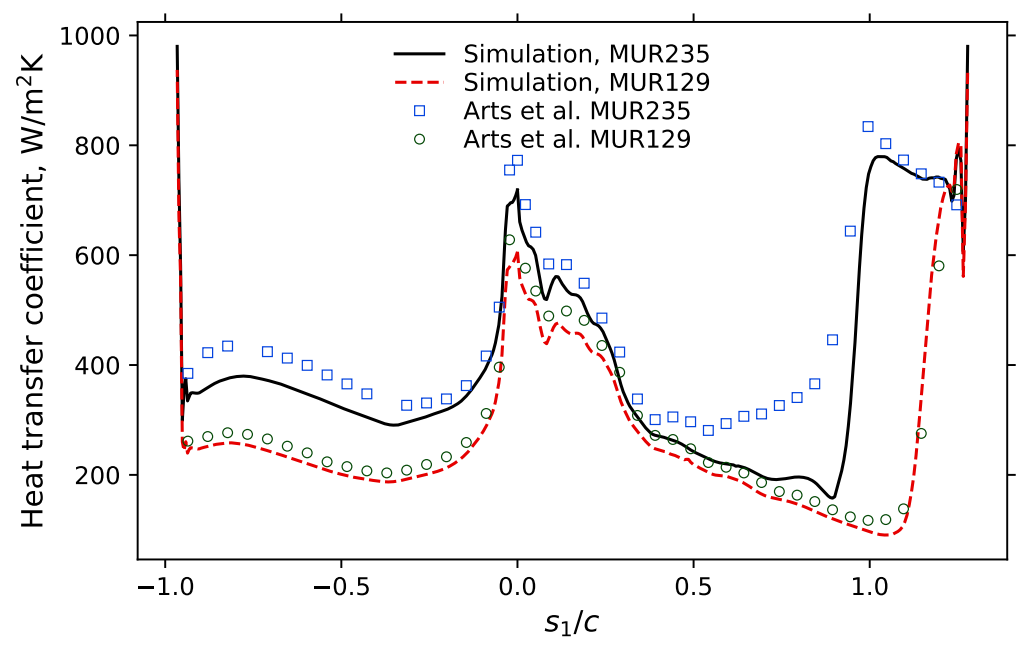

Figure 3: Heat transfer coefficient along the blade surface compared to the experimental data of Arts et al. [1].

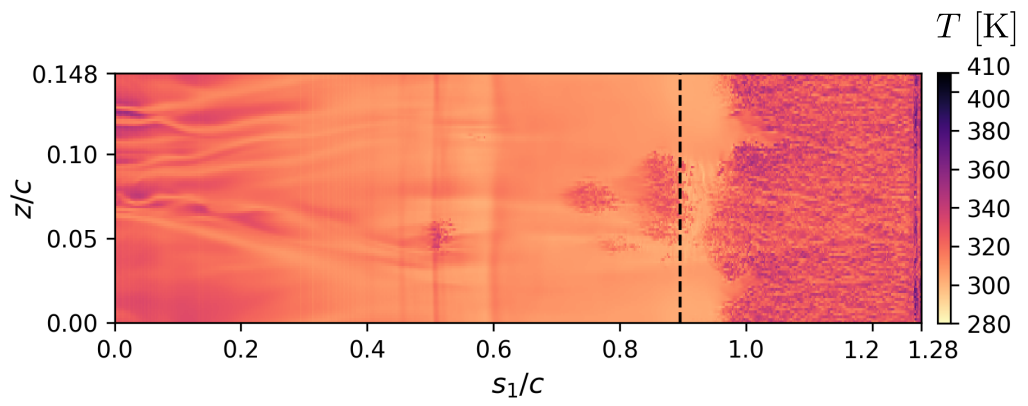

Figure 4: Instantaneous field of temperature $T$ on the offset surface P1 defined in section 2.4, namely an offset surface at a fixed distance from the suction-side blade wall, in the MUR235 test case. The vertical line represents the approximate shock location.

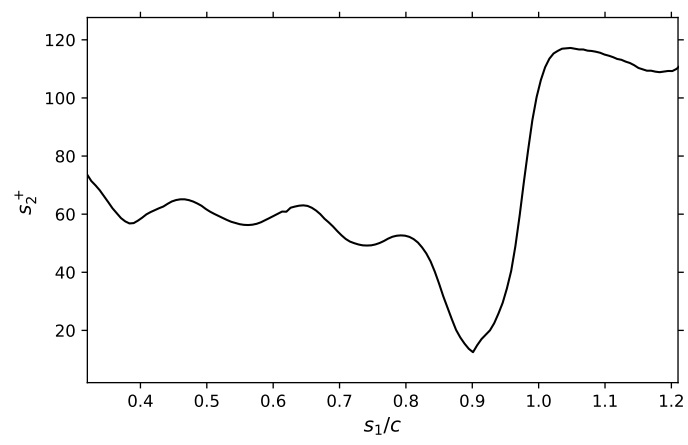

Figure 5: Distance of the offset surface P1 from the suction-side blade wall, in wall units. 

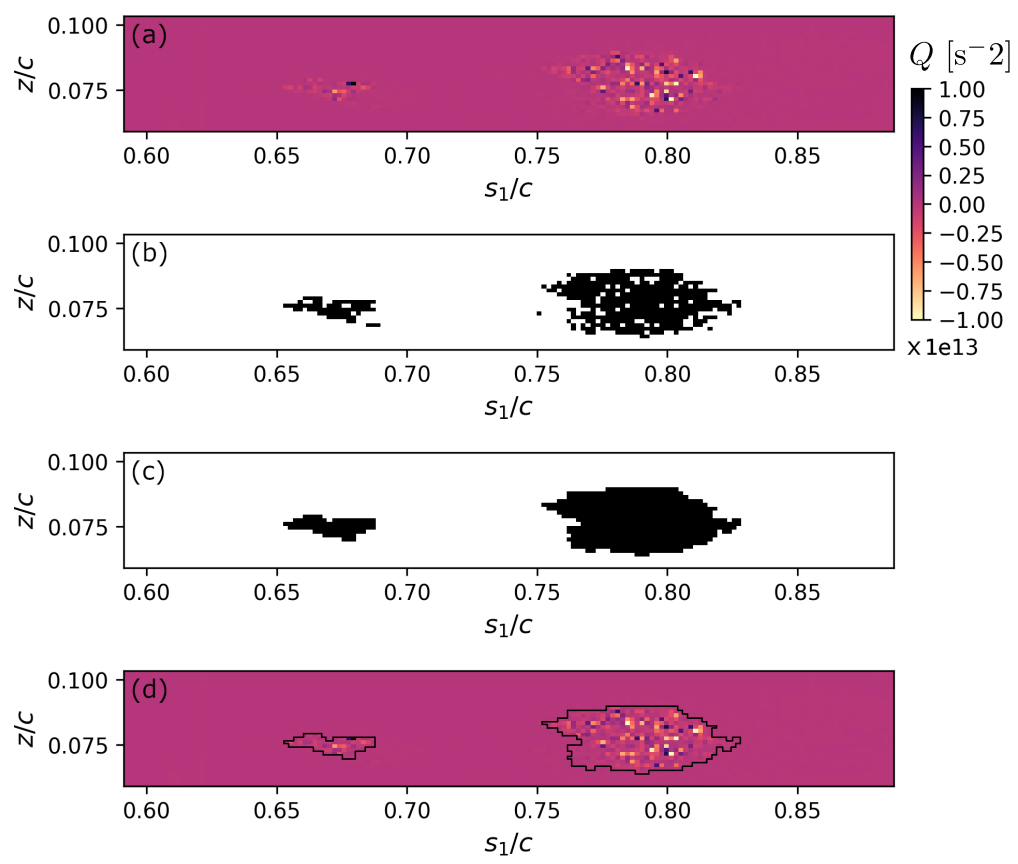

Figure 6: Identification of the turbulent spots: (a) instantaneous field of $Q$ criterion interpolated on the offset surface $\mathrm{P} 1, \mathrm{~s}^{-2}$; (b) location of values above the threshold; (c) filtering and smoothing to obtain contiguous regions without holes; and (d) edges of the identified regions superimposed the original image.

0.45); a transitional region, downstream, in which turbulent spots may appear $\left(s_{1} / c=0.45-\right.$ $0.9)$; and a fully turbulent region after the shock $\left(s_{1} / c>0.9\right)$. In this section, the production and growth of the turbulent spots observed in the transitional region are characterised. First, the effect of the acoustic waves on the production of the observed turbulent spots is addressed. Second, the dynamics of the turbulent spots is examined to confirm their physical relevance. The objectives are to guarantee the reliability of the numerical flow features and provide a basis for future improvements on the MUR235 test case prediction. The influence of the observed turbulent spots on the shock/boundary-layer interaction is then investigated in more detail.

\subsection{Turbulent-spot production}

The production, spanwise and streamwise growth, convection and eventual merging of several turbulent spots (T.S.) on the suction side of the blade may be seen in Figure 7(a)-(d). Numerical simulations of the MUR129 test case of Arts et al. [1], which has similar operating conditions but a lower inlet turbulence intensity of $0.8 \%$, have not identified turbulent spots on the suction side of the blade $[10,21,67]$. This shows that freestream turbulence is crucial for the production of turbulent spots. Turbulent spot production is in this case most likely to occur through a bypass mechanism following the classification of Mayle [40]. In light of Figure 2, 4 and 7(a)-(d), three phenomena may be presumed to contribute to the destabilisation of the flow at the onset of the transitional boundary layer: the presence of elongated streaks generated at the leading edge of the blade; the local freestream turbulence; and the impingement on the suction side of acoustic waves (A.W.) as seen in Figure 2.

In order to verify whether there is a causal relationship between the generation of acoustic waves at the trailing edge of the blade and the production of turbulent spots on the suction side of the blade, a numerical experiment is performed in which acoustic waves are suppressed using the dynamic mode tracking and control (DMTC) of Queguineur et al. [55]. The method is based on a two-part procedure. First, acoustic waves are identified using an operator-based [73] second-order band-stop filter centred on the frequency of the acoustic wave generation. Second, a relaxation term is added to the flow governing equations in order to create a feedback control loop that forces 

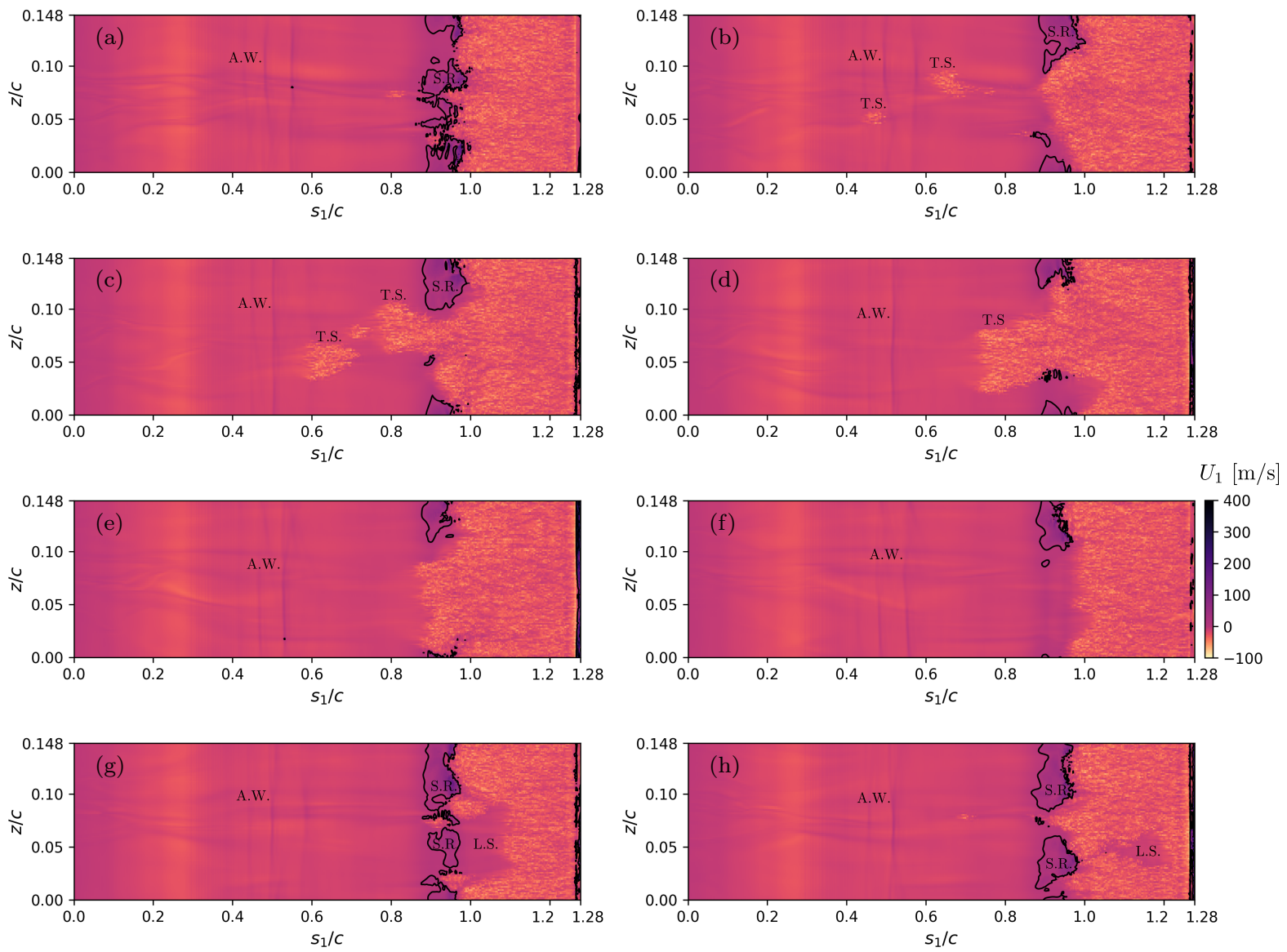

Figure 7: Instantaneous field of tangential velocity $U_{1}$ on the offset surface $\mathrm{P} 1$, for eight evenly spaced timesteps, from $t / t_{c}=0.0318$ (a) to $t / t_{c}=0.592(\mathrm{~h}), \mathrm{m} / \mathrm{s}$. The contour line is the level set $U_{1}=0$. The vertical line represents the approximate shock location. Denoted are acoustic waves (A.W.), separation region (S.R.), turbulent spots (T.S.) and laminar spots (L.S.).

the flow to avoid the selected frequency. Figure 8 compares the pressure field obtained using this numerical procedure to the reference pressure field obtained without control. The control is seen to successfully damp the acoustic-wave generation at the trailing edge of the blade and severely reduces the vortex shedding in the wake, as both phenomena are associated with the same fundamental frequency. Figure 9 shows that despite the removal of acoustic waves, the presence of turbulent spots on the suction-side may still be observed in the simulation with dynamic control of acoustic waves, demonstrating that acoustic waves are not necessary for the production of turbulent spots. The main mechanism of spot production is thus likely associated with the destabilisation of the vortical structures generated at the leading edge of the blade, which is typical of boundary layer transition under a freestream vortical forcing [28, 78, 45], as investigated by Zhao and Sandberg [80] for another operating point.

\subsection{Growth dynamics of the turbulent spots}

The spatiotemperal representations given in Figure 10 at $z=L_{z} / 4$ as a function of the curvilinear blade coordinate (Figure $10(\mathrm{a})$ ) and at $s_{1} / c=0.89$ as a function of the spanwise coordinate (Figure 10(b)) show the intermittent production of turbulent spots. The periodic incidence of acoustic waves generated at the trailing edge and reflected by the blade surface is also clearly visible on these figures. Note that the production of turbulent spots seems to coincide with the occurrence of events coming from the leading edge, although not all of these events lead to a turbulent spot. Figure 11 provides a more global spatiotemperal representation taking into account all spots 
(a)

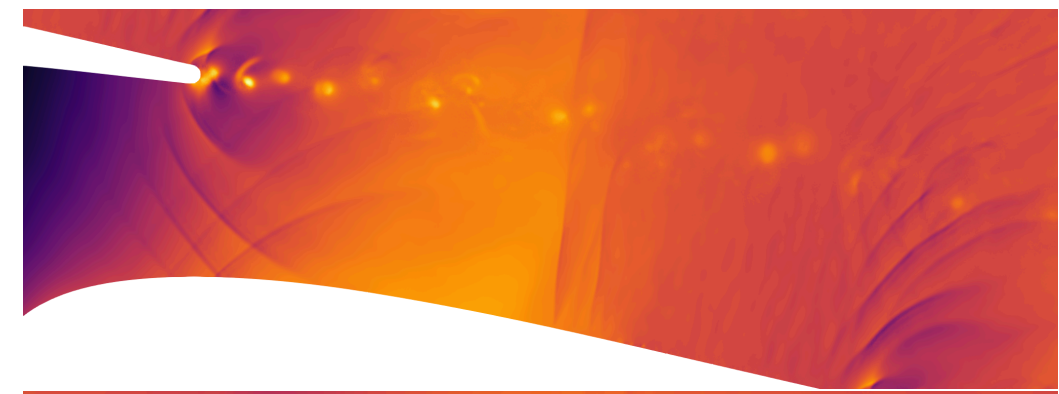

(b)

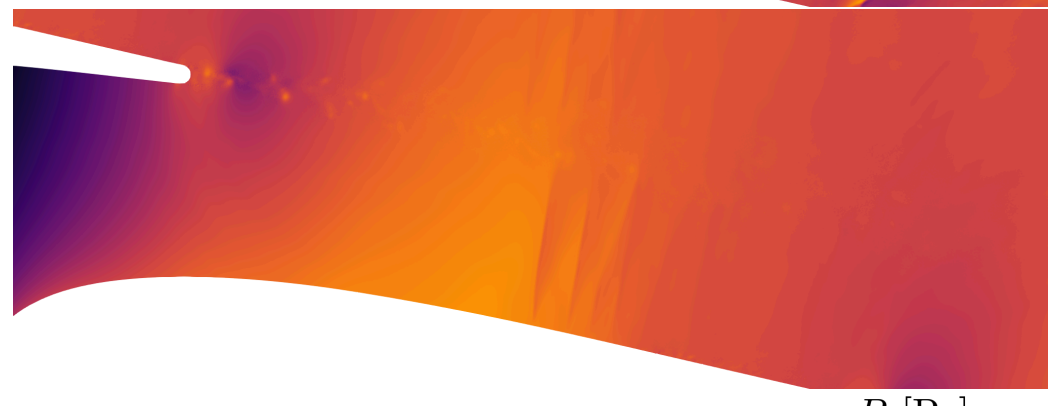

$6.5 e+04 \quad 1 e+5 \quad 1.6 e+05$

Figure 8: Instantaneous field of pressure on the plane $z / c=0.037$ without control (a) and with dynamic control of acoustic waves (b).

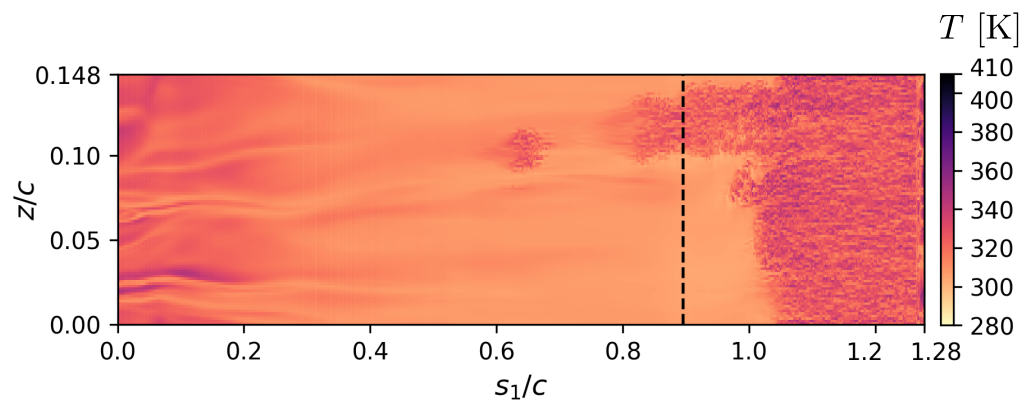

Figure 9: Instantaneous field of temperature $T$ on the offset surface P1 with dynamic control of acoustic waves. The vertical line represents the approximate shock location.

in the spanwise direction as a function of the curvilinear blade coordinate. This representation suggests that the turbulent spots tend to be produced in intermittent groupings of two or three spots temporally very close to each other. This behaviour, previously observed in Murphree et al. [42], is not consistent with an assumption of spot-formation independency and might be related to the occurrence of large freestream turbulent events, destabilising the flow nearly simultaneously at several locations.

The development of the transitional region is characterised by the intermittency $\Gamma$, defined as the statistical frequency for which a given location is turbulent. The distribution of the intermittency along the blade surface is given in Figure 12. Numerically, the intermittency is observed to rise along the transitional region to a level of $10 \%$, before the effect of the shock impingement is felt. Following the shock $\left(s_{1} / c>0.9\right)$, the intermittency increases very sharply to near fully turbulent levels, suggesting breakdown to turbulence. A fully turbulent condition $\Gamma=1$ is however not obtained due to parts of the turbulent regions misrepresented as laminar by the detection method on the one hand but also due to an observed partial laminarisation of the post-shock area following the arrival of the spots, as discussed in section 3.3. According to the transition model of Narasimha [43] under the assumption of concentrated breakdown on a flat plate, the intermittency 


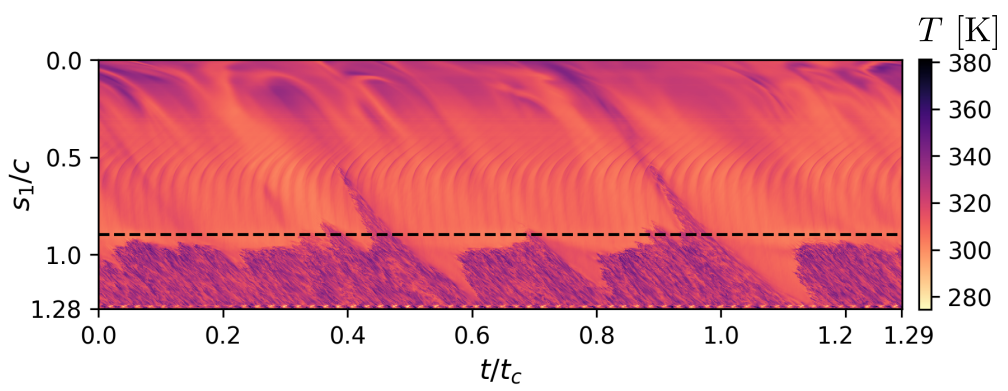

(a)

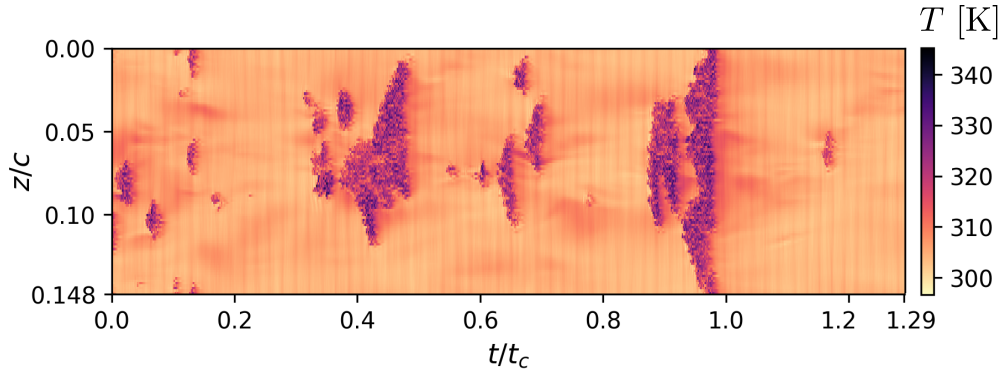

(b)

Figure 10: Spatiotemporal representation of temperature on the intersection of the offset surface P1 with the plane $z / c=0.037$ (a) and $s_{1} / c=0.89(\mathrm{~b}), \mathrm{K}$. The horizontal line represents the approximate average shock location.

distribution should be of the form

$$
\Gamma\left(s_{1}\right)=1-\exp \left(-\frac{0.412}{\lambda_{t}^{2}}\left(H\left(s_{1}-s_{t}\right)\right)^{2}\right),
$$

where $H$ is the Heaviside step function, $s_{t}$ is the location of the transition onset and $\lambda_{t}$ characterises the length of the transition. A least-square fit of this distribution over the numerical results on the transitional region yields $s_{t} / c=0.45$, in agreement with the visual inspection of Figure 11, and $\lambda_{t} / c=1.01$, which implies an end of transition beyond the suction side length. With the same model, the transition length may be related to the rate of turbulent-spot production $n$ and the dynamics of the spot growth: upstream, downstream and spanwise growth rate of the spot boundaries and interaction between spots, such that,

$$
\lambda_{t}=0.642 \sqrt{\frac{U_{\infty}}{n \sigma}},
$$

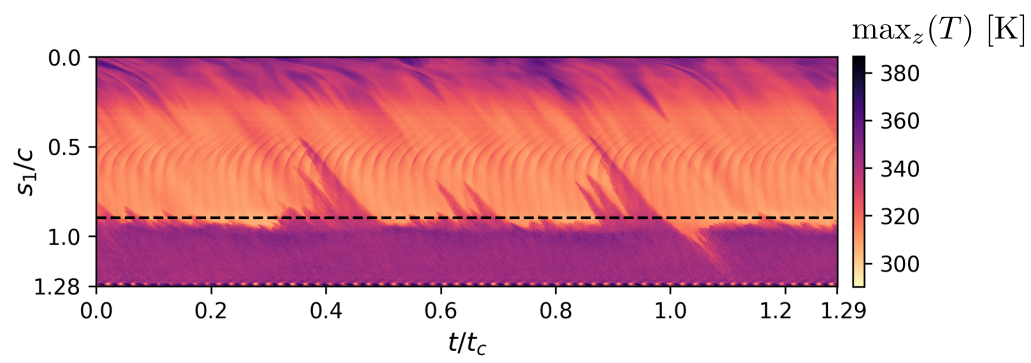

Figure 11: Spatiotemporal representation of the maximum temperature along the spanwise direction on the offset surface P1, K. The horizontal line represents the approximate average shock location. 


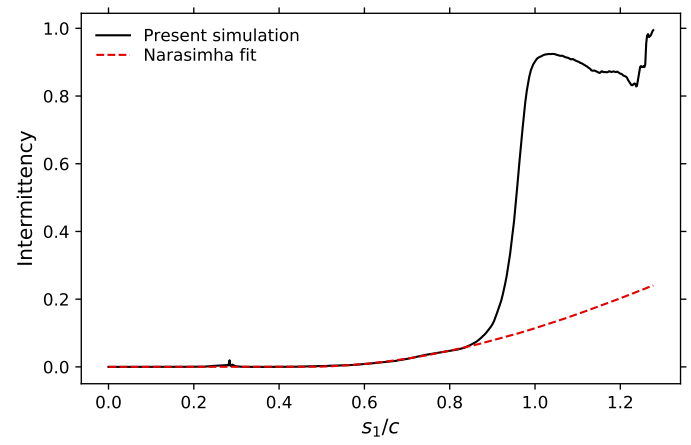

Figure 12: Intermittency along the blade surface and fit to Narasimha distribution (9) over the transitional region.

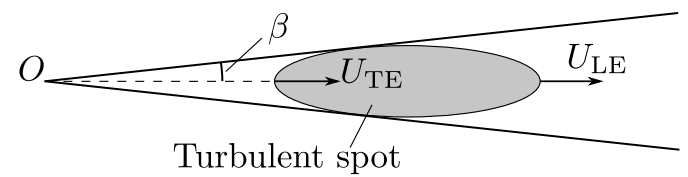

Figure 13: Leading-edge velocity $U_{\mathrm{LE}}$, trailing-edge velocity $U_{\mathrm{TE}}$ and spreading half-angle $\beta$ of a turbulent spot produced at the location $O$.

with $U_{\infty}$ the tangential velocity at the edge of the boundary layer and $\sigma$ a dimensionless spotpropagation parameter [15], modelled as $\sigma=U_{\infty}\left(1 / U_{T E}-1 / U_{L E}\right) \tan (\beta)$, where $U_{T E}$ is the velocity of the spot trailing edge (upstream boundary), $U_{L E}$ is the velocity of the spot leading edge (downstream boundary) and $\beta$ is the spot spreading half-angle, represented schematically in Figure 13.

To determine numerically the parameters of equation (10), the boundaries of 19 turbulent spots have been tracked using the identification method presented in section 2.4. Note however that given the favorable pressure gradient and surface curvature in the transitional region, it is not clear that the growth rates of spots at different locations are comparable. Indeed, the growth of turbulent spots may not be explained solely by classical turbulent entrainment and involves in addition the destabilisation of the surrounding flow [18]. Convex curvature and positive pressure gradients have stabilising effects that decrease the spanwise growth rate of turbulent spots, while concave curvature and adverse pressure gradients have the opposite effect [31, 69]. The cooling effect of the blade wall temperature may also have an influence on the turbulent spot growth [56]. Figure 14 reports the leading-edge and trailing-edge velocity of the spots scaled by the local freestream velocity along the blade surface, as well their spreading half-angle. While there is a lot of variability between the values obtained for each individual spot, no clear pattern can be discerned with regard to the dependency on the curvilinear abscissa $s_{1}$ in spite of the aforementioned limits. The spotgrowth parameters averaged over each turbulent spot are given in Table 3 and compared to the correlations of Van den Eynde and Steelant [74], which takes into account the stabilising effect of the Mach number [33] but disregards the effect of the pressure gradient, curvature or temperature:

$$
\begin{gathered}
\frac{U_{\mathrm{LE}}}{U_{\infty}} \approx 0.82+0.017 M a_{\infty}, \\
\frac{U_{\mathrm{TE}}}{U_{\infty}} \approx 0.52+0.025 M a_{\infty}, \\
\frac{\beta}{\beta_{0}} \approx \exp \left(-0.28 M a_{\infty}\right), \\
\sigma \approx F_{0}\left(1.8-0.8 \tanh \left(8 M a_{\infty}\right)\right)\left(\frac{U_{\infty}}{U_{\mathrm{TE}}}-\frac{U_{\infty}}{U_{\mathrm{LE}}}\right) \tan (\beta),
\end{gathered}
$$

with $M a_{\infty}$ the Mach number at the edge of the boundary layer and $\beta_{0}=11.3^{\circ}$ the spot-spreading 


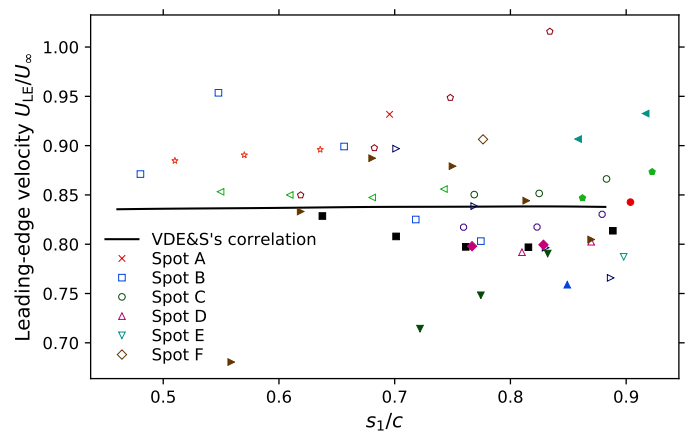

(a)

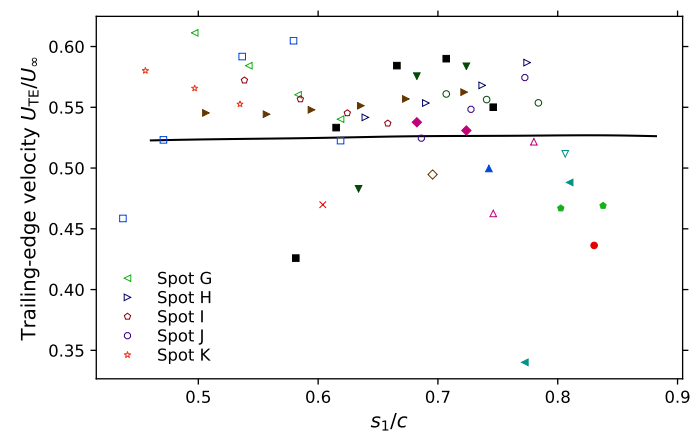

(b)

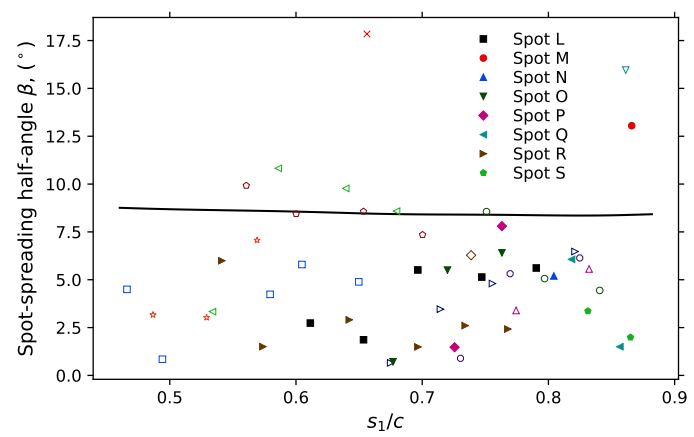

(c)

Figure 14: Numerical estimation of the leading-edge velocity (a), trailing-edge velocity (b) and spreading half-angle (c) of the spots along the blade surface. The correlations of Van den Eynde \& Steelant [74] is also given.

half-angle at the incompressible limit. A good agreement between the numerical results and the correlations is obtained for the leading-edge velocity and the trailing-edge velocity, suggesting a limited influence of the favorable pressure gradient, the wall temperature and curvature. Previous studies on a flat plate $[8,62]$ are consistent with this result as the leading-edge velocity was found to remain a constant fraction of the local freestream velocity under a mild pressure gradient. With regard to the spot-spreading half-angle, a significantly lower value than implied by the correlation is obtained, despite the dispersion of the results. This might be related to the stabilising effect of the convex curvature and of wall temperature reducing the spanwise growth of turbulent spots. The longitudinal interaction between spots can also contribute to the decrease of spanwise growth since turbulent spots tend to occur in intermittent groupings in our simulation [34]. Given the overall agreement between the numerical results and the correlations of Van den Eynde and Steelant [74], the observed turbulent spots can confidently be regarded as physical. The following section therefore details their intermittent impact on the shock/boundary-layer interaction.

VDE\&S's Averaged numerical results correlation (standard deviation)

\begin{tabular}{lcc}
\hline$U_{\mathrm{LE}} / U_{\infty}$ & 0.84 & $0.84(0.06)$ \\
$U_{\mathrm{TE}} / U_{\infty}$ & 0.52 & $0.53(0.05)$ \\
$\beta,\left(^{\circ}\right)$ & 8.5 & $5.4(3.6)$ \\
$\sigma$ & 0.097 & $0.066(0.048)$
\end{tabular}

Table 3: Comparison of the averaged numerical spot leading-edge velocity, trailing-edge velocity and spreading half-angle with the correlations of Van den Eynde \& Steelant [74]. 


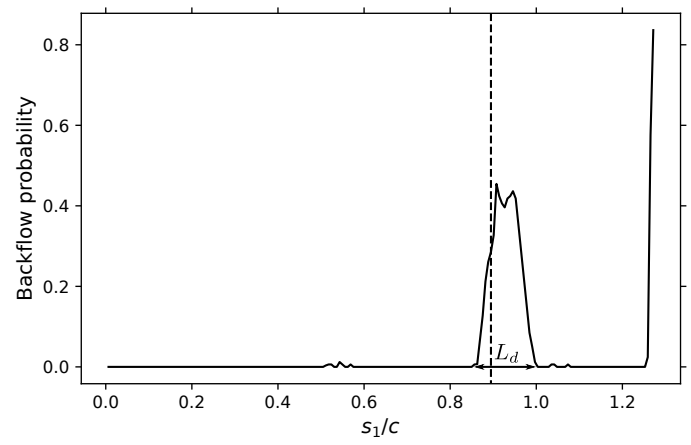

Figure 15: Statistical frequency of backflow along the blade surface at the spanwise location $z / c=0.037$ (i.e. at a quarter of the spanwise blade length). The vertical line represents the approximate average shock location.

\subsection{Shock/boundary-layer interaction}

The shock is indeed seen to have a complex and unsteady interaction with the transitional boundary layer. When the boundary layer is fully laminar without turbulent spots, an instantaneous region of separation (S.R.) is formed following the shock (Figure 7(a)). The destabilisation of this separation bubble explains the rapid transition to turbulence downstream of the shock, as investigated in previous studies $[59,30,63,23,25]$. In the following, instantaneous separation is considered congruent with local backflow, that is the occurrence of negative tangential velocity within the boundary layer, as in Quadros and Bernardini [54], such that the boundary-layer detachment can be characterised by the backflow probability $B$. According to the classification of Simpson [70], a boundary-layer detachment with a backflow probability $B \geq 1 \%$ is termed as an incipient separation (IS), $B \geq 20 \%$ as an intermittent transitory detachment (ITD) and $B \geq 50 \%$ as a transitory detachment (TD). The distribution of the backflow probability obtained from the simulation along the blade surface is given in Figure 15. The width of the separation region is $L_{d} / c=0.13$. The peak backflow probability is 0.45 , which corresponds to an intermittent transitory detachment. Note that the boundary-layer detachment is not massive as backflow is not observed in the mean. The unsteady nature of the flow separation is in a large part due to the influence of the turbulent spots on the shock/boundary-layer interaction.

Indeed, if an instantaneous region of separation is present, the turbulent spots "tunnel" through the separation bubble which remains locally attached (Figure 7(c),(d) and Figure 16(a),(c)). A similar effect has been encountered by Krishnan and Sandham [35] on a flat-plate surface. This behaviour suggests that the shock is too weak to induce separation on a turbulent boundary layer with the present flow conditions. Note that in such a problem, the pressure required for incipient separation, $P_{\text {inc }}$, may be assessed using the free-interaction theory of Chapman et al. [6]:

$$
\frac{P_{\mathrm{inc}}}{P_{\infty}}=1+C \gamma M_{\infty}^{2} \sqrt{\frac{2 C_{f}}{\sqrt{M_{\infty}^{2}-1}}}
$$

with $C_{f}$ the skin friction coefficient and $C$ a constant that depends on the state of the incoming boundary layer. The required pressure ratio in a laminar interaction is estimated using $C=1$ [24] along with the skin friction coefficient at the onset of the shock/boundary-interaction, located at $s_{1} / c=0.45$. The required pressure ratio for a turbulent interaction is estimated using $C=3[2]$ as well as the skin friction coefficient in the turbulent area downstream of the shock, around $s_{1} / c=1.1$. This implies incipient separation at a pressure ratio of 1.1 for a laminar boundary layer and 2.6 for a turbulent boundary layer. The pressure ratio of 1.2 obtained in the simulation between the transitional and turbulent regions, can thus be expected to be insufficient for the detachment of the turbulent boundary layer within the spots, while the laminar boundary layer has a lower resistance to the adverse pressure gradient created by the shock [11]. Following the arrival of the spot, localised regions of laminar flow (laminar spots, L.S.) appear in the post-shock region, that is otherwise fully turbulent (Figure $7(\mathrm{~g})-(\mathrm{h})$ and Figure $16(\mathrm{e})-(\mathrm{f})$ ). This may be 

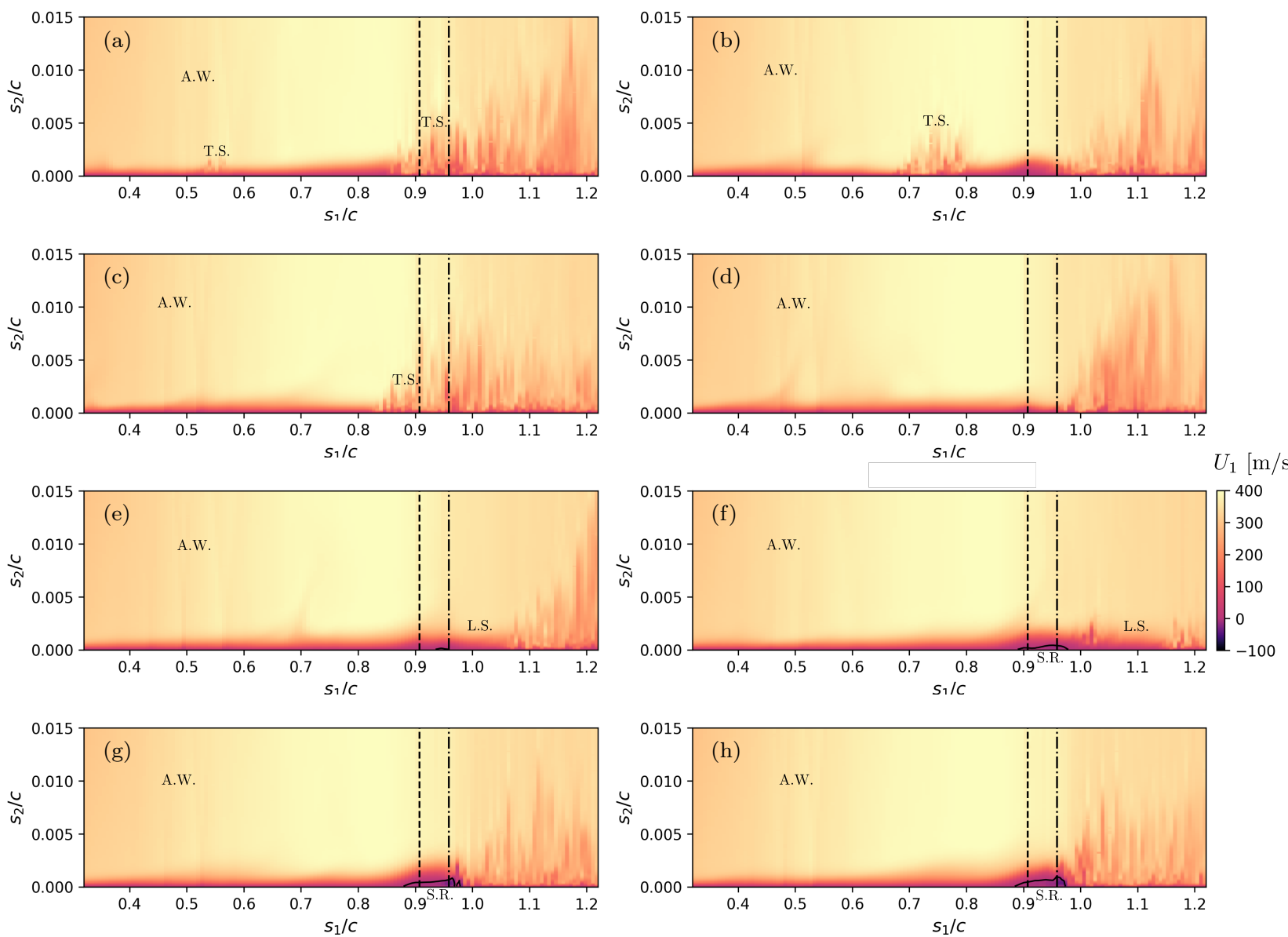

Figure 16: Instantaneous field of tangential velocity $U_{1}$ on the plane $z / c=0.037$, for eight evenly spaced timesteps, from $t / t_{c}=0.0377$ (a) to $t / t_{c}=0.651(\mathrm{~h}), \mathrm{m} / \mathrm{s}$. The contour line is the level set $U_{1}=0$. The dashed vertical line represents the approximate shock location. The dot-dashed vertical line is the plane $s_{1} / c=0.96$ featured in Figure 17. Denoted are acoustic waves (A.W.), separation region (S.R.), turbulent spots (T.S.) and laminar spots (L.S.).

explained by the relatively long establishment time of the separation bubble, the destabilisation thereof being required to ensure transition to turbulence. Indeed, the structure of turbulent spots involves a calmed region behind the spot with a very low receptiveness to disturbances, thus remaining laminar and resisting to separation [66]. It is observed in our simulations that the laminar spots have a negative growth rate due to the growth of surrounding turbulent areas and usually vanish before reaching the trailing edge of the blade. The spatiotemperal representations given in Figure 17 at the curvilinear abscissa $s_{1} / c=0.96$ show the intermittent reattachment and detachment of the boundary layer depending on the incidence of turbulent spots. The boundary layer remains laminar and attached for a short period after the passage of a turbulent spot as there is a clear delay to the redetachment of the boundary layer. A duration of around 0.1 characteristic time without incoming spots is required for the establishment of the separation bubble. Thus, a larger rate of turbulent-spot production reduces the transient separation of the flow at the location of the shocks.

\section{Effect of the intermittency}

At this stage of the analysis, multiple elements support the plausibility of the flow physics observed in the MUR235 test case. However, the consequence of the identified transient flow phenomena on the mean flow properties is still unclear. In the following, efforts are made to quantify 


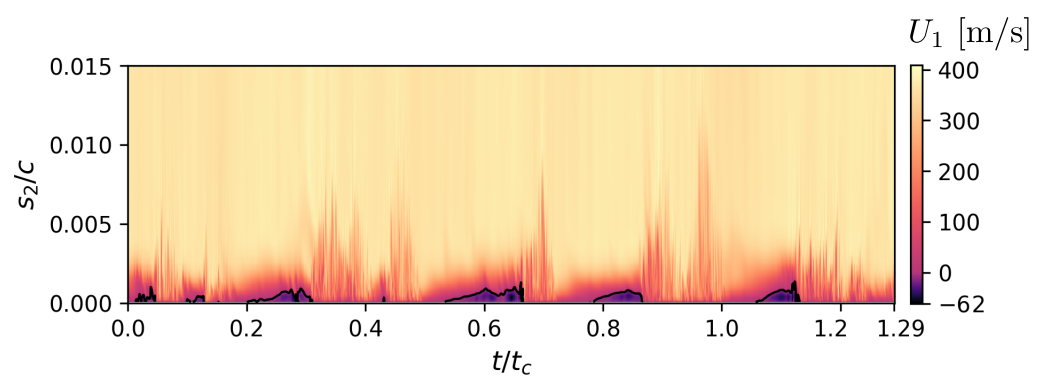

Figure 17: Spatiotemporal representation of tangential velocity on the intersection of the planes $s_{1} / c=0.96$ (see dot-dashed line in Figure 16) and $z / c=0.037$ (i.e. at a quarter of the spanwise blade length), $\mathrm{m} / \mathrm{s}$.

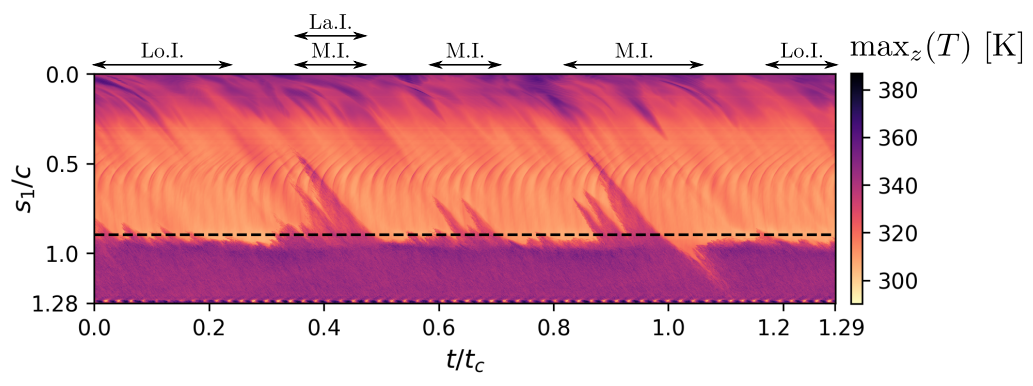

Figure 18: Averaging durations corresponding to the large-intermittency (La.I.), mediumintermittency (M.I.) and low-intermittency (Lo.I.) cases superimposed the spatiotemporal representation of the maximum temperature along the spanwise direction on the offset surface P1, K. The horizontal line represents the approximate average shock location.

this aspect and allow relevant and constructive conclusions to be drawn from such simulations. First, a measure of the influence of the turbulent spots on the wall shear stress and heat transfer is proposed. A specific focus is then placed on kinetic energy exchanges.

\subsection{Wall shear stress and heat transfer}

The production of turbulent spots has a large effect on the wall heat transfer on the suction side of the blade. This effect is thereafter characterised through the comparison of conditional averages based on the presence of turbulent spots in the transitional region. The identification method presented in section 2.4 is used for this purpose. This direct detection approach is preferred to methods based on probability-density functions [64] as a clear separation into two peaks for laminar and turbulent flow regions is not observed.

For the analysis, three cases have been defined, as reported in Figure 18: the low-intermittency case (Lo.I.), which selects durations with an almost fully laminar boundary layer upstream of the shock; the medium-intermittency case (M.I.), which comprises all major turbulent-spot production events; and the large-intermittency case (La.I), which corresponds to a single turbulent-spot event. The temporal windows chosen to define the low-, medium- and large-intermittency cases are associated with an averaging duration of $0.35,0.47$ and 0.12 characteristic times respectively. The intermittency level corresponding to each case is given in Figure 19.

Figure 20 gives the effect of the intermittency in the transitional region on the shape factor, which characterises the state of the boundary layer. The shape factor is defined as $H=\delta^{*} / \theta$, where

$$
\delta^{*}=\int_{0}^{\delta}\left(1-\frac{\langle\rho\rangle\left\langle U_{1}\right\rangle}{\rho_{\infty} U_{\infty}}\right) d s_{2}
$$




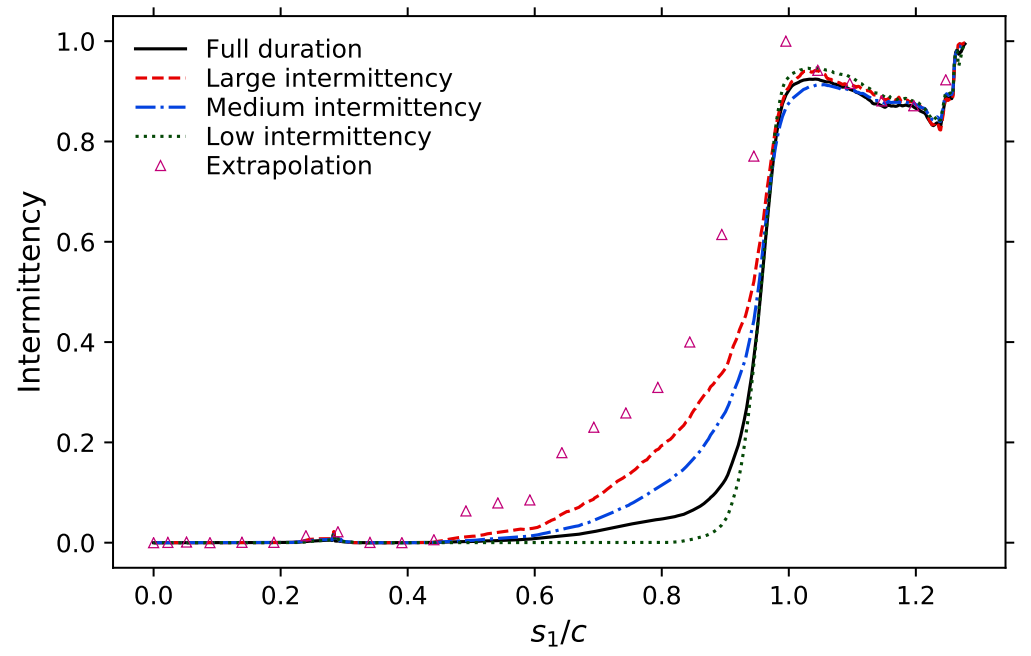

Figure 19: Intermittency along the blade surface using the full averaging duration or partial averaging durations with a large or small number of spots. The 'extrapolation' is an estimation of the intermittency required to obtain the experimental heat transfer coefficient of Arts et al. [1], as described in the text.

is the displacement thickness while

$$
\theta=\int_{0}^{\delta}\left(1-\frac{\left\langle U_{1}\right\rangle}{U_{\infty}}\right) \frac{\langle\rho\rangle\left\langle U_{1}\right\rangle}{\rho_{\infty} U_{\infty}} d s_{2},
$$

is the momentum thickness, where $\rho_{\infty}$ is the density at the edge of the boundary layer. Note that to perform the integration, boundary layer thickness $\delta$ is defined using the vorticity criterion of Michelassi et al. [41]. Namely, the edge of the boundary layer satisfies

$$
\langle\omega\rangle\left(s_{2}=\delta\right)=\left\langle\omega_{\min }\right\rangle+\frac{\left\langle\omega_{\max }\right\rangle-\left\langle\omega_{\min }\right\rangle}{100},
$$

where $\langle\omega\rangle=\langle\|\nabla \times \boldsymbol{U}\|\rangle$ is the mean vorticity magnitude and $\omega_{\min }$ and $\omega_{\max }$ are respectively the minimum and maximum values of the vorticity magnitude in the cross-section. A slow decrease of the shape factor in the transitional region $\left(s_{1} / c=0.45-0.9\right)$ is obtained in the mediumintermittency and large-intermittency cases, which is consistent with the more pronounced transitional region. In the region around the shock $\left(s_{1} / c \approx 0.9\right)$, the shape factor peak is decreased in the medium-intermittency case as well as the large-intermittency case while it is increased in the low-intermittency case, since the presence of spots in the transitional region decreases the likelihood of boundary-layer separation. Finally, note that care should be taken in interpreting the conditional averages downstream of the shock since it depends on the state of the transitional boundary layer at a previous time.

The intermittency of the transitional region is expected to have a significant effect on the skin friction (Figure 20) and heat transfer at the wall (Figure 21). The medium-intermittency and large-intermittency cases have a significantly larger wall shear stress or heat flux in the transitional region $\left(s_{1} / c=0.45-0.9\right)$, narrowing the gap with the reference experimental results. This shows that a large part of the differences between the experiment of Arts et al. [1] and simulations can be explained by an underestimation of the rate of turbulent-spot production. Effectively, the present large-eddy simulation does not capture accurately the destabilisation of the boundary layer on the suction side of the blade, leading to a lower overall intermittency in the transitional region. On this basis, an estimation of the intermittency level required to reproduce the heat transfer coefficient of Arts et al. [1], assuming a linear heat-transfer/intermittency dependence, is given in Figure 19. The estimated experimental intermittency should be above $40 \%$ before the effect of the shock is felt, i.e. roughly five times the numerical value for the present simulation. Despite this discrepancy, the last section of our work focuses on exploiting the present database to highlight the impact of 


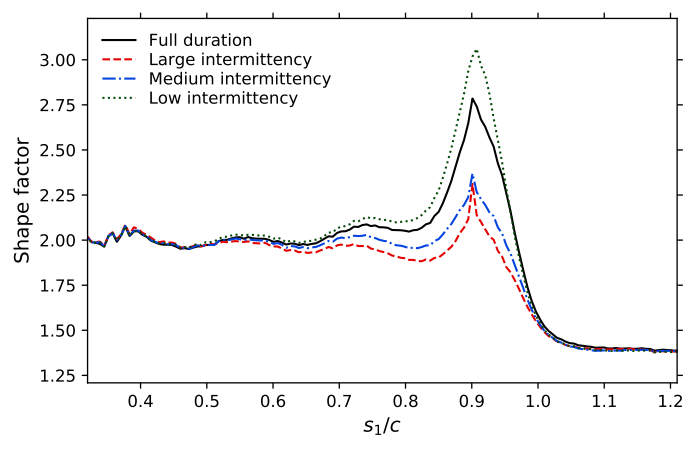

(a)

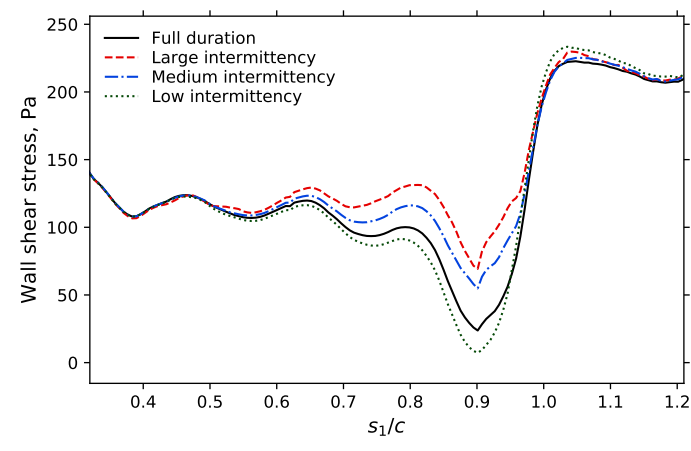

(b)

Figure 20: Shape factor (a) and wall shear stress (b) along the blade surface for three intermittency levels.

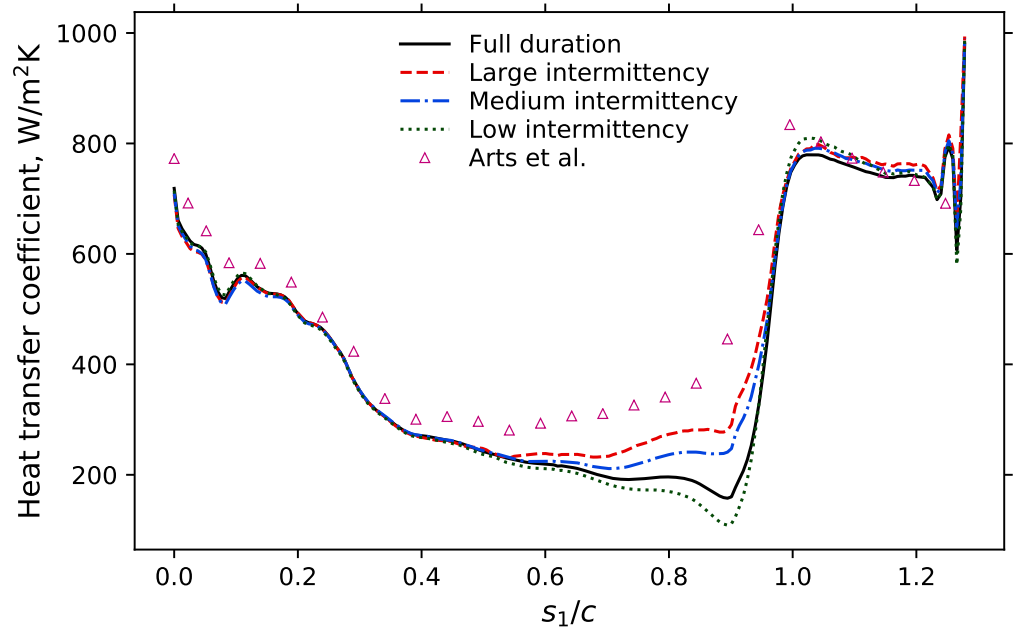

Figure 21: Heat transfer coefficient along the suction-side blade surface using the full averaging duration or partial averaging durations with a large or small number of spots.

the turbulent spots on the kinetic energy balance within the boundary layer and ultimately the losses.

\subsection{Kinetic energy exchanges}

This section investigates the effect of the intermittency on kinetic energy exchanges, and in particular the dissipation of kinetic energy. The analysis is performed using the semi-local scaling which is a suitable scaling for kinetic energy exchanges in variable-property wall-bounded flows [47, $48,13,14]$. With the semi-local scaling, denoted $\left(^{*}\right)$, turbulence statistics are scaled by a linear combination of the local density, viscosity and the semi-local friction velocity, defined as $U_{\tau}^{*}=$ $\sqrt{\left(\mu_{\omega} /\langle\rho\rangle\right)\left(\partial_{2}\left\langle U_{1}\right\rangle\right)_{\omega}}$, where $\omega$ denotes wall values. In particular, the wall-normal coordinate $s_{2}$ is scaled by $\langle\nu\rangle / U_{\tau}^{*}$, velocity components by $U_{\tau}^{*}$ and kinetic energy exchanges by $\langle\rho\rangle U_{\tau}^{* 4} /\langle\nu\rangle$. The profile of tangential velocity with the semi-local scaling is given in Figure 22 at three locations on the suction side. As discussed in section 4.1, one of the main effects of a larger intermittency in the transitional region is an increase in wall shear stress. This results in a larger scaled tangential velocity outside the boundary layer. The effect is salient near the shock, since the transient flow separation greatly decreases the wall shear stress in the absence of turbulent spots. The overlap of the three conditional averages in the turbulent region is indicative of the time convergence of the average.

With regard to kinetic energy exchanges, the transport equation of kinetic energy may be 
expressed in the current large-eddy simulation formalism as

$$
\frac{\partial\langle\rho\rangle\{K\}}{\partial t}+\frac{\partial\langle\rho\rangle\left\{U_{j} K\right\}}{\partial x_{j}}+\frac{\partial\left\langle P U_{j}\right\rangle}{\partial x_{j}}-\frac{\partial\left\langle\Sigma_{i j} U_{i}\right\rangle}{\partial x_{j}}=\left\langle P \frac{\partial U_{j}}{\partial x_{j}}\right\rangle-\left\langle\Sigma_{i j} \frac{\partial U_{i}}{\partial x_{j}}\right\rangle,
$$

with $K=\frac{1}{2} U_{i} U_{i}$ the instantaneous kinetic energy per unit mass and $\{\cdot\}=\langle\rho \cdot\rangle /\langle\rho\rangle$ the Favre average. Viscous dissipation $\langle\mathcal{E}\rangle=\left\langle\Sigma_{i j} \partial_{j} U_{i}\right\rangle$, which represents the irreversible rate of transformation of kinetic energy into internal energy, is directly related to entropy generation as it may be expressed as the product of viscous entropy generation rate and absolute temperature. The profiles of viscous dissipation near the suction-side wall are given in Figure 23. In the transitional region, a larger intermittency level increases the scaled dissipation at the wall but decreases the scaled dissipation around $s_{2}^{*}=15$. Near $s_{2}^{*}=7$, the scaled dissipation is invariant with respect to intermittency. In other words, the amplitude of dissipation at this location scales as $\langle\rho\rangle U_{\tau}^{* 4} /\langle\nu\rangle$ and may be predicted during the transition process from the evolution of the wall shear stress. This behaviour persists throughout the transitional region until the effect of the shock if felt. In the low-intermittency case, dissipation is not maximum at the wall but near the shock $\left(s_{1} / c=0.89\right)$ as a secondary peak emerges near $s_{2}^{*}=10$. This secondary peak dissapears further downstream in the turbulent region. Note that the profiles of dissipation encompasses both resolved viscous effects and subgrid-scale effects. The subgrid-scale activity [20], which can be approximated following Celik et al. [5] as the fraction of subgrid-scale viscosity

$$
\varsigma=\frac{\mu_{\mathrm{sgs}}}{\mu(T)+\mu_{\mathrm{sgs}}},
$$

is a measure of the magnitude of subgrid-scale dissipation compared to total dissipation. On the suction side of the blade, subgrid-scale dissipation is small up to $s_{2}^{*} \approx 50$, after which it becomes predominant near and downstream of the shock (Figure 24). The regions of large dissipation discussed above are thus mainly due to resolved viscous effects as dissipation is very small for $s_{2}^{*} \gtrsim 50$.

Further understanding of the dissipation profiles may be obtained by decomposing viscous dissipation into a turbulent part $\varepsilon$ related to the turbulent motion and a mean part $\overline{\mathcal{E}}$ related to the mean motion from the similar decomposition of kinetic energy. In the Favre averaging formalism [16], turbulent kinetic energy is defined as $k=\frac{1}{2} u_{i}^{\prime \prime} u_{i}^{\prime \prime}$ and the associated mean kinetic energy as $\bar{K}=\frac{1}{2}\left\{U_{i}\right\}\left\{U_{i}\right\}$, where $u_{i}^{\prime \prime}=U_{i}-\left\{U_{i}\right\}$ is the Favre fluctuation of velocity. In the current large-eddy simulation formalism, the transport equation of turbulent kinetic energy may be expressed as,

$$
\begin{aligned}
\frac{\partial\langle\rho\rangle\{k\}}{\partial t}+ & \frac{\partial\langle\rho\rangle\left\{U_{j} k\right\}}{\partial x_{j}}+\frac{\partial\left\langle p^{\prime} u_{j}^{\prime}\right\rangle}{\partial x_{j}}-\frac{\partial\left\langle\sigma_{i j}^{\prime} u_{i}^{\prime}\right\rangle}{\partial x_{j}}= \\
& -\langle\rho\rangle\left\{u_{i}^{\prime \prime} u_{j}^{\prime \prime}\right\} \frac{\partial\left\{U_{i}\right\}}{\partial x_{j}}-\left\langle u_{j}^{\prime \prime}\right\rangle \frac{\partial\langle P\rangle}{\partial x_{j}}+\left\langle p^{\prime} \frac{\partial u_{j}^{\prime}}{\partial x_{j}}\right\rangle+\left\langle u_{i}^{\prime \prime}\right\rangle \frac{\partial\left\langle\Sigma_{i j}\right\rangle}{\partial x_{j}}-\left\langle\sigma_{i j}^{\prime} \frac{\partial u_{i}^{\prime}}{\partial x_{j}}\right\rangle,
\end{aligned}
$$

and the transport equation of mean kinetic energy as,

$$
\begin{aligned}
\frac{\partial\langle\rho\rangle\{\bar{K}\}}{\partial t}+ & \frac{\partial\langle\rho\rangle\left\{U_{j} \bar{K}\right\}}{\partial x_{j}}+\frac{\partial\langle P\rangle\left\langle U_{j}\right\rangle}{\partial x_{j}}-\frac{\partial\left\langle\Sigma_{i j}\right\rangle\left\langle U_{i}\right\rangle}{\partial x_{j}}= \\
& \langle\rho\rangle\left\{u_{i}^{\prime \prime} u_{j}^{\prime \prime}\right\} \frac{\partial\left\{U_{i}\right\}}{\partial x_{j}}+\left\langle u_{j}^{\prime \prime}\right\rangle \frac{\partial\langle P\rangle}{\partial x_{j}}+\langle P\rangle \frac{\partial\left\langle U_{j}\right\rangle}{\partial x_{j}}-\left\langle u_{i}^{\prime \prime}\right\rangle \frac{\partial\left\langle\Sigma_{i j}\right\rangle}{\partial x_{j}}-\left\langle\Sigma_{i j}\right\rangle \frac{\partial\left\langle U_{i}\right\rangle}{\partial x_{j}},
\end{aligned}
$$

where $\boldsymbol{u}^{\prime}=\boldsymbol{U}-\langle\boldsymbol{U}\rangle, p^{\prime}=P-\langle P\rangle$ and $\sigma_{i j}^{\prime}=\Sigma_{i j}-\left\langle\Sigma_{i j}\right\rangle$ are the fluctuating part of velocity, pressure and viscous shear stress respectively [27]. The production is classically defined as the term $\langle\mathcal{P}\rangle=-\langle\rho\rangle\left\{u_{i}^{\prime \prime} u_{j}^{\prime \prime}\right\} \partial_{j}\left\{U_{i}\right\}$, and represents an interaction between turbulent kinetic energy and mean kinetic energy. The turbulent dissipation is $\langle\varepsilon\rangle=\left\langle\sigma_{i j}^{\prime} \partial_{j} u_{i}^{\prime}\right\rangle$ and the mean dissipation $\langle\overline{\mathcal{E}}\rangle=\left\langle\Sigma_{i j}\right\rangle \partial_{j}\left\langle U_{i}\right\rangle$. Note that this decomposition is not unique since other representation of the energy exchanges are possible [12]. Numerically, the mean dissipation is computed following Wheeler et al. [75] as,

$$
\langle\overline{\mathcal{E}}\rangle=\left\langle\mu(T)+\mu_{\mathrm{sgs}}\right\rangle\left(\frac{\partial\left\langle U_{i}\right\rangle}{\partial x_{j}} \frac{\partial\left\langle U_{i}\right\rangle}{\partial x_{j}}+\frac{\partial\left\langle U_{j}\right\rangle}{\partial x_{i}} \frac{\partial\left\langle U_{i}\right\rangle}{\partial x_{j}}-\frac{2}{3} \frac{\partial\left\langle U_{k}\right\rangle}{\partial x_{k}} \frac{\partial\left\langle U_{j}\right\rangle}{\partial x_{j}}\right),
$$




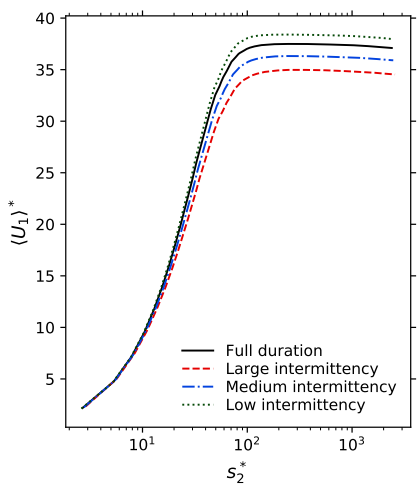

(a)

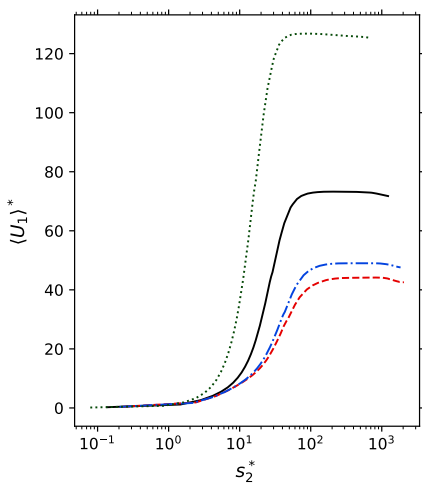

(b)

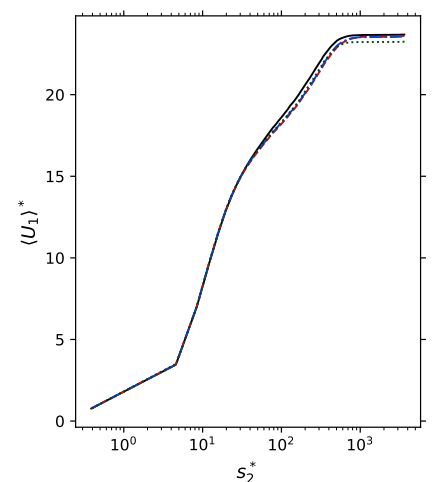

(c)

Figure 22: Profiles of tangential velocity $U_{1}$ at three locations on the suction side of the blade with the semi-local scaling: $s_{1} / c=0.70(\mathrm{a}), s_{1} / c=0.89(\mathrm{~b})$ and $s_{1} / c=1.09(\mathrm{c})$.

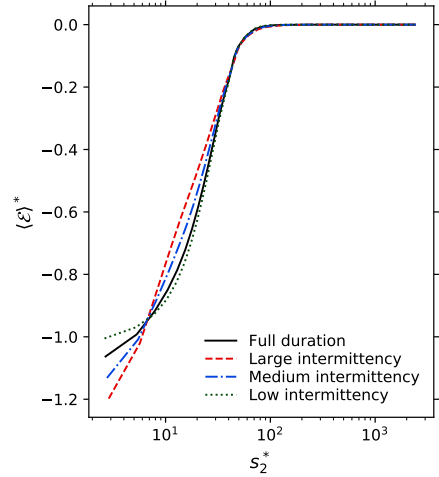

(a)

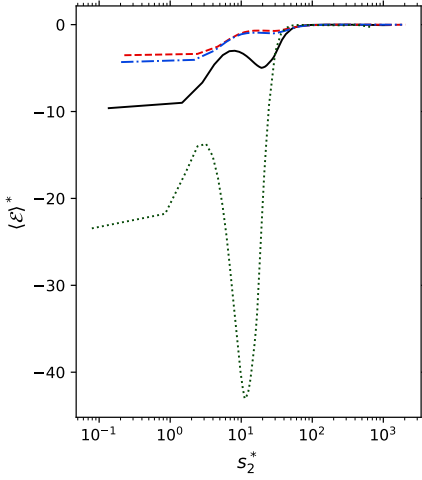

(b)

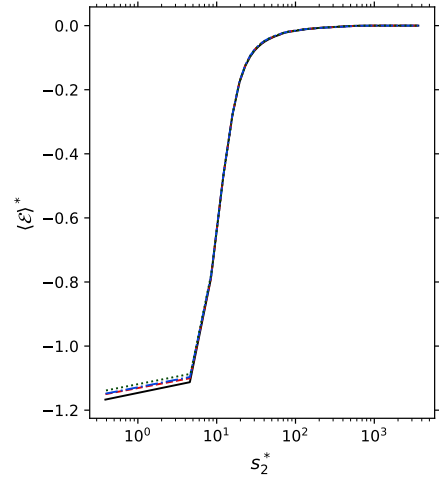

(c)

Figure 23: Profiles of dissipation at three locations on the suction side of the blade with the semilocal scaling: $s_{1} / c=0.70(\mathrm{a}), s_{1} / c=0.89(\mathrm{~b})$ and $s_{1} / c=1.09(\mathrm{c})$.

and the turbulent dissipation as $\langle\varepsilon\rangle=\langle\mathcal{E}\rangle-\langle\overline{\mathcal{E}}\rangle$, neglecting the effect of viscosity fluctuations [27, 13].

Profiles of turbulence production, turbulent dissipation and mean dissipation are given in Figure 25. With the semi-local scaling, the peak of production in the transitional region is increased and shifted towards the wall as the intermittency is raised. The peak of turbulent dissipation, at the wall, also rises sharply with intermittency. However, the peak of mean dissipation is unchanged. Instead, a decrease of the scaled mean dissipation around $s_{2}^{*}=15$ is observed for large intermittencies. The increase in turbulent dissipation and the decrease in mean dissipation cancel out near $s_{2}^{*}=7$, which explains the invariant behaviour of the total dissipation at this location. Around the shock, the mean dissipation peaks near $s_{2}^{*}=10$ and is negligible at the wall due to the flow separation, whereas the turbulent dissipation remains maximum at the wall. The peaks of turbulent dissipation and mean dissipation correspond to the two local maxima of total dissipation in Figure 23. The distribution of turbulence production and dissipation integrated over the boundary layer height is finally plotted in Figure 26. The total production of turbulent kinetic energy remains larger than its dissipation over the entire blade surface. In particular, there is a large excess of production around the shocks, as found in Xiutao et al. [77]. There are two local production peaks, located directly above the onset $\left(s_{1} / c=0.89\right)$ and termination $\left(s_{1} / c=0.97\right)$ of the region of transient separation. The comparison of the low- and large-intermittency cases shows that the first peak is not present for a fully laminar incoming boundary layer and thus is associated with the arrival of turbulent spots. This is confirmed by the two-dimensional representation given in Figure 27. In the large-intermittency case, the production starts increasing in 


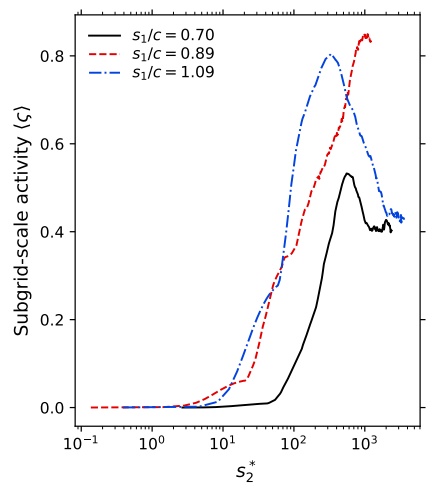

Figure 24: Profiles of mean subgrid-scale activity at three locations on the suction side of the blade with the semi-local scaling: $s_{1} / c=0.70 s_{1} / c=0.89$ and $s_{1} / c=1.09$.

the transitional region due to the incidence of turbulent spots and peaks around the shocks. This peak is not present in the low-intermittency case, in which production peaks slightly upstream the transition to a turbulent boundary layer. In both cases, the classical behaviour of production and dissipation in a turbulent wall-bounded flow [51] is obtained downstream of the shock, with a peak of production at a fixed distance $s_{2}^{*} \approx 13$ from the wall (Figure 25). All these findings underline the complexity of the energetic mechanisms that occurs in such a transient flow. Evaluations of existing RANS closures on such observations are clearly of interest for further improvements.

\section{Conclusion}

The numerical simulation of the transonic high-pressure linear nozzle blade cascade VKI-LS89 under the test case MUR235 shows a complex interaction between shocks, wake vortex shedding, boundary layer and acoustic waves. Although existing, the available large-eddy simulations of the blade underestimate the wall heat flux along the suction-side boundary layer. The present study identifies the presence of turbulent spots in this region and argues using conditional averaging of flow statistics based on the intermittency in the transitional region that the discrepancy may be explained by an insufficient rate of turbulent-spot production in the simulations. The leading-edge and trailing-edge velocities of the observed turbulent spots are well predicted by correlations based solely on the Mach number, which provide some confidence in the reliability of the turbulent-spot physics. The arrival of turbulent spots modifies the shock/boundary-layer interaction since it forces the boundary layer to remain locally attached, whereas detachment is observed in the absence of spots. Laminar spots may then be observed in the otherwise turbulent region downstream of the shock due to the stabilising effect of the spots and the establishment time of the separation bubble. The intermittency level upstream of the shock is thus expected to have a strong influence on this interaction. Kinetic energy exchanges also display an intermittent behaviour in the transitional region. The occurrence of a large number of spots is associated with a large increase of viscous dissipation and turbulence production in the transitional region. In particular, a large amount of production is in that case observed near the shock location. The intermittency level required to reproduce the experimental heat transfer has been estimated to be above $40 \%$ before the effect of the shock is felt, which is significantly larger than the value obtained in the simulation. Future improvements on the MUR235 test case should ensure that the averaging duration takes into account the duration and frequency of the intermittent flow events. Furthermore, the production of vortical structures at the leading edge of the blade and their impact on the transition process should be investigated in more detail. 


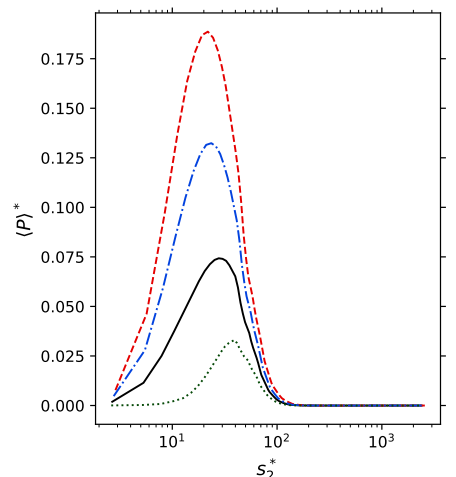

(a)

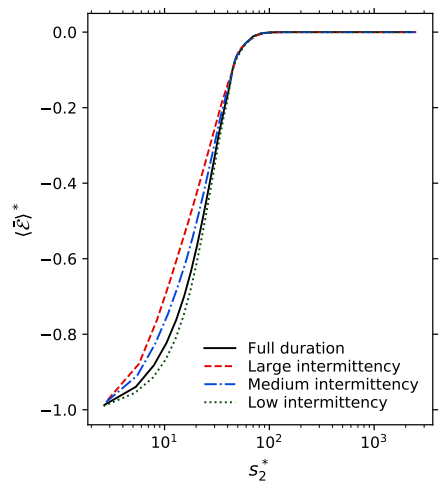

(d)

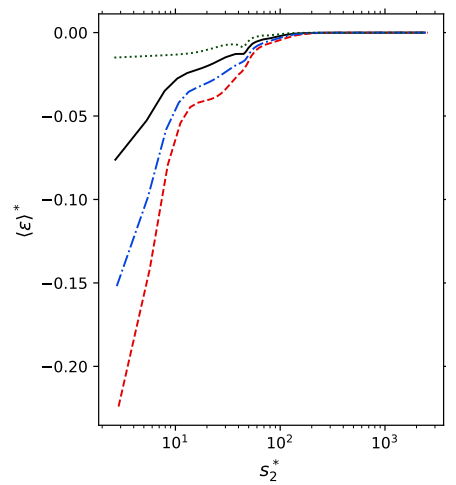

(g)

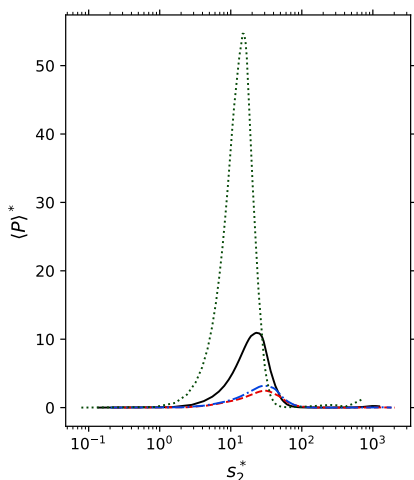

(b)

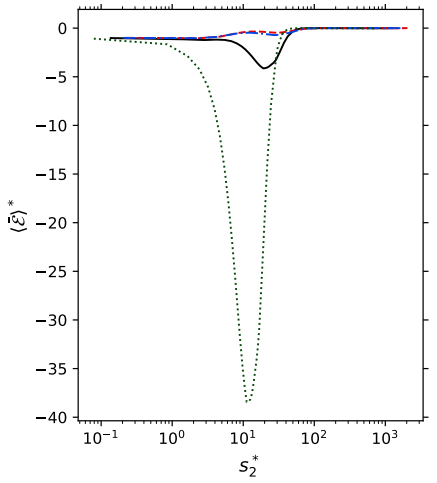

(e)

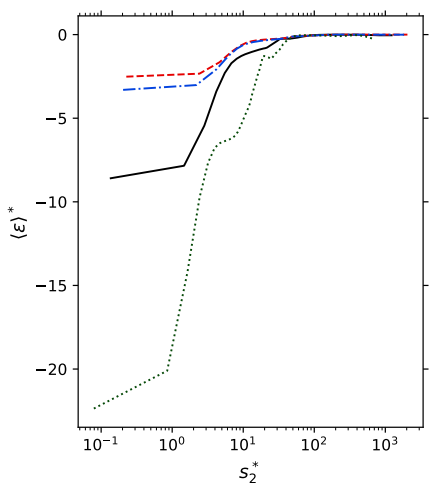

(h)

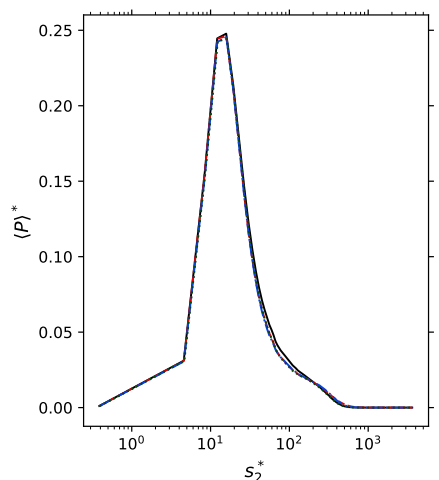

(c)

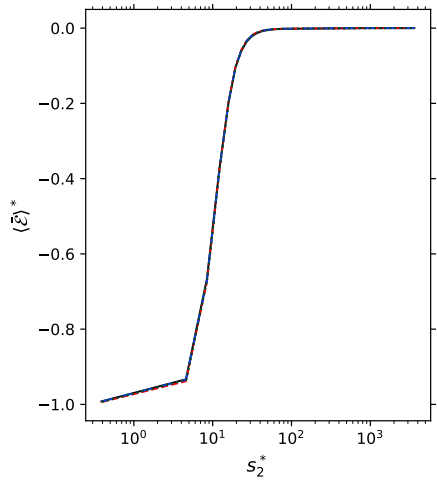

(f)

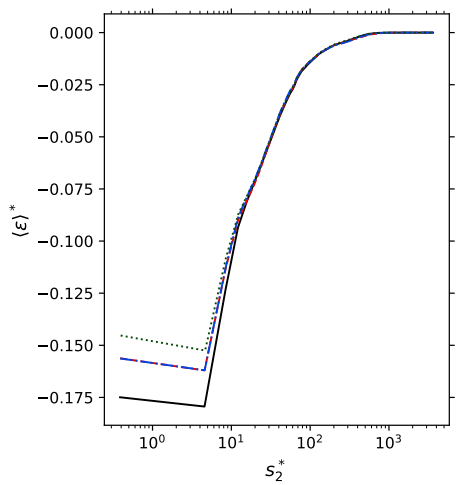

(i)

Figure 25: Profiles of production (a, b, c), mean dissipation (d, e, f) and turbulent dissipation (g, $\mathrm{h}, \mathrm{i}$ ) at three locations on the suction side of the blade with the semi-local scaling: $s_{1} / c=0.70$ (a, $\mathrm{d}, \mathrm{g}), s_{1} / c=0.89(\mathrm{~b}, \mathrm{e}, \mathrm{h})$ and $s_{1} / c=1.09(\mathrm{c}, \mathrm{f}, \mathrm{i})$. 


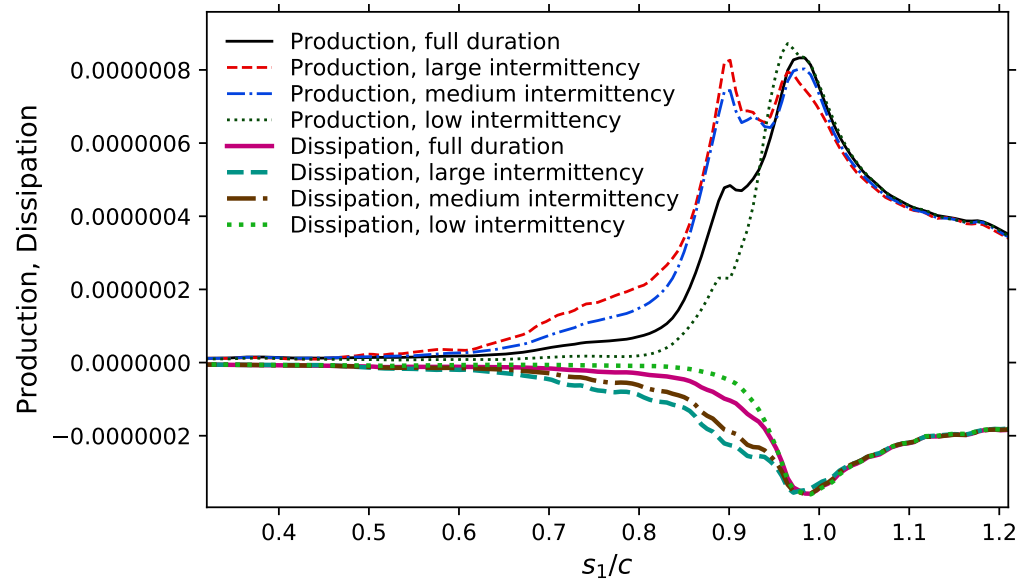

Figure 26: Production and turbulent dissipation integrated over the boundary layer height along the suction-side blade surface using the full averaging duration or partial averaging durations with a large or small number of spots, scaled by $\rho_{\text {inlet }} U_{\text {inlet }}^{4} /\left(\nu_{\text {inlet }} c\right)$.

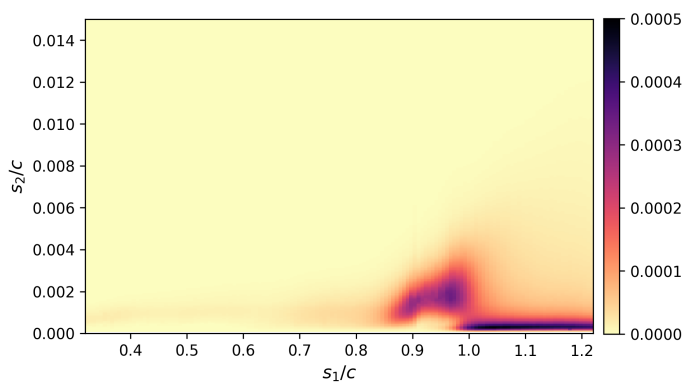

(a)

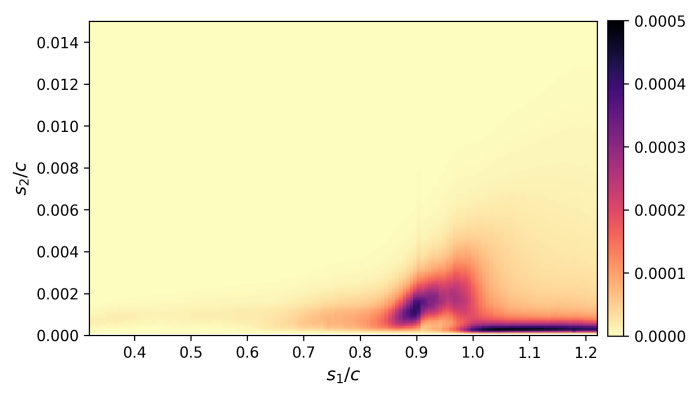

(c)

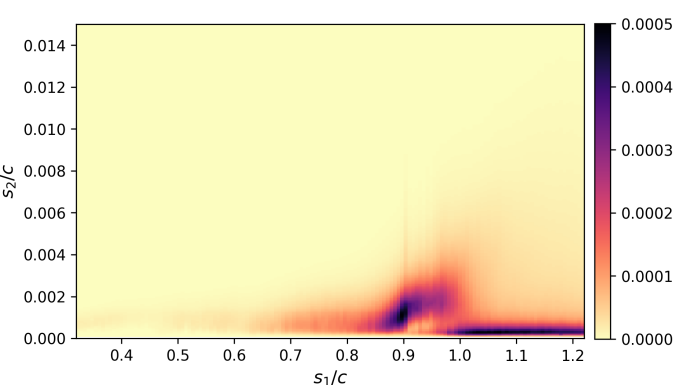

(b)

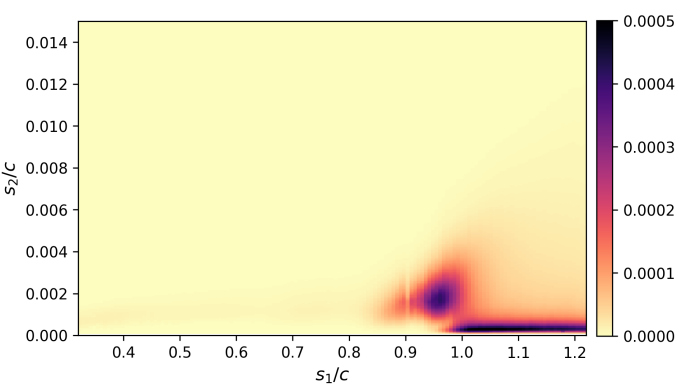

(d)

Figure 27: Turbulence production on the suction side of the blade using the full averaging duration (a) or the partial averaging durations corresponding to the large-intermittency case (b), the medium-intermittency case (c) and the low-intermittency case (d), scaled by $\rho_{\text {inlet }} U_{\text {inlet }}^{4} / \nu_{\text {inlet }}$. 


\begin{tabular}{lrrc} 
& \multicolumn{1}{c}{ M1 } & \multicolumn{1}{c}{ M2 } & M3 \\
\hline Total number of cells & $65.7 \times 10^{6}$ & $213.0 \times 10^{6}$ & $586.8 \times 10^{6}$ \\
Total number of prisms & $8.1 \times 10^{6}$ & $89.5 \times 10^{6}$ & $269.4 \times 10^{6}$ \\
Total number of nodes & $28.9 \times 10^{6}$ & $135.4 \times 10^{6}$ & $386.2 \times 10^{6}$
\end{tabular}

Table 4: Mesh parameters of the meshes M1, M2 and M3.

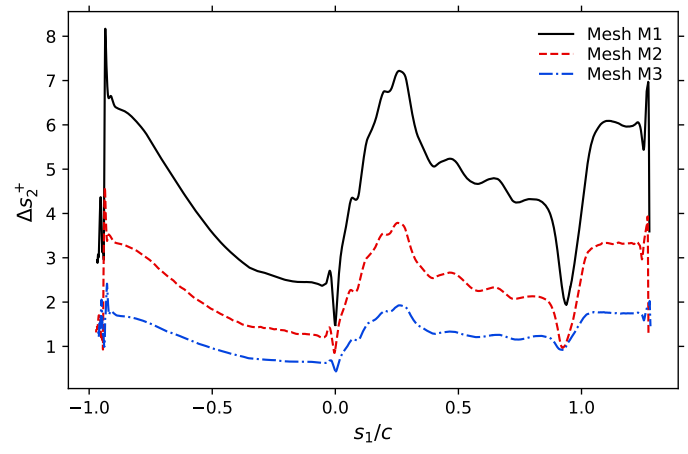

Figure 28: Height of the first cell off the wall along the blade surface for the meshes M1, M2 and M3, in wall units.

\section{Acknowledgments}

This work was granted access to the computer resources of CINES and TGCC under the allocation 2019-A0062A06074 made by GENCI. D. Dupuy has been funded by the RTRA-STAE foundation (Réseau thématique de recherche avancée Sciences et technologies pour l'aéronautique et l'espace).

\section{Data availability}

The data that support the findings of this study are available from the corresponding author upon reasonable request.

\section{A Effect of the mesh refinement}

For the MUR235 test case, three hybdrid meshes have been considered, hereafter referred to as M1, M2 and M3. The parameters of the three meshes are given in Table 4. The meshes M2 and M3 are more refined than the coarse mesh M1 in both the near-wall region and the freestream. Specific refinements are also performed in the trailing edge region. Figure 28 compares the distribution of the height of the first cell off the wall along the blade surface with the three meshes. The mean heat transfer coefficient obtained using three meshes is compared to the experimental data of Arts et al. [1] in Figure 29. There is an overall agreement between the simulations and the experiment for the shape of the heat transfer distribution. On the pressure side of the blade, the heat transfer prediction is improved with the meshes M2 and M3 compared to the mesh M1. On the suction side, the location of the shock-induced boundary layer transition is well predicted the three simulations. Downstream of the shock, the heat transfer level is in agreement with the experiment using the mesh M3 but is underestimated with the meshes M1 and M2. Upstream of the shock, the simulations predict accurately the heat transfer in the strongly accelerating region near the leading edge. Further downstream however, the three simulations predict a heat transfer decrease from $s_{1} / c=0.5$ to 0.9 which is not present in the experimental data. As discussed in in section 4.1, a large part of the discrepancy between the experimental results and the simulations can be attributed to a larger rate of turbulent-spot production. Turbulent spots have been observed with the three 


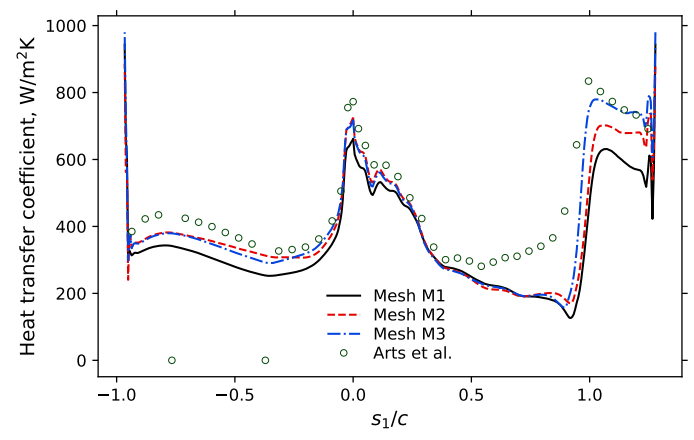

Figure 29: Heat transfer coefficient along the blade surface for the meshes M1, M2 and M3, compared to the experimental data of Arts et al. [1].

meshes, but with a insufficient intermittency in the transitional region. Since the turbulent region downstream of the shock is well resolved with the mesh M3, it is reasonable to assume that the turbulent spots, which have many features of fully turbulent boundary layers [4, 58], are also well described by the simulation.

\section{References}

[1] T. Arts, M. L. De Rouvroit, and A. W. Rutherford. Aero-thermal investigation of a highly loaded transonic linear turbine guide vane cascade. von Karman Institute for Fluid Dynamics, 1990.

[2] H. Babinsky and J. K. Harvey. Shock wave-boundary-layer interactions. Cambridge University Press, New York, 2011.

[3] R. Bhaskaran and S. K. Lele. Large eddy simulation of free-stream turbulence effects on heat transfer to a high-pressure turbine cascade. Journal of Turbulence, (11):N6, 2010.

[4] B. Cantwell, D. Coles, and P. Dimotakis. Structure and entrainment in the plane of symmetry of a turbulent spot. Journal of Fluid Mechanics, 87(4):641-672, 1978.

[5] I. B. Celik, Z. N. Cehreli, and I. Yavuz. Index of resolution quality for large eddy simulations. Journal of Fluids Engineering, 127(5):949-958, 2005.

[6] D. R. Chapman, D. M. Kuehn, and H. K. Larson. Investigation of separated flows in supersonic and subsonic streams with emphasis on the effect of transition. NACA Rept., 1356, 1958.

[7] R. V. Chima. Application of the k-omega turbulence model to quasi-three-dimensional turbomachinary flows. Journal of Propulsion and Power, 12(6):1176-1179, 1996.

[8] J. P. Clark, T. V. Jones, and J. E. LaGraff. On the propagation of naturally-occurring turbulent spots. Journal of Engineering Mathematics, 28(1):1-19, 1994.

[9] O. Colin and M. Rudgyard. Development of high-order Taylor-Galerkin schemes for LES. Journal of Computational Physics, 162(2):338-371, 2000.

[10] E. C. Collado Morata, N. Gourdain, F. Duchaine, and L. Y. M. Gicquel. Effects of free-stream turbulence on high pressure turbine blade heat transfer predicted by structured and unstructured LES. International Journal of Heat and Mass Transfer, 55(21-22):5754-5768, 2012.

[11] J. M. Délery. Shock wave/turbulent boundary layer interaction and its control. Progress in Aerospace Sciences, 22(4):209-280, 1985.

[12] D. Dupuy, A. Toutant, and F. Bataille. Equations of energy exchanges in variable density turbulent flows. Physics Letters A, 382(5):327-333, 2018.

[13] D. Dupuy, A. Toutant, and F. Bataille. Turbulence kinetic energy exchanges in flows with highly variable fluid properties. Journal of Fluid Mechanics, 834:5-54, 2018.

[14] D. Dupuy, A. Toutant, and F. Bataille. Effect of the Reynolds number on turbulence kinetic energy exchanges in flows with highly variable fluid properties. Physics of Fluids, 31(1):015104, 2019.

[15] H. W. Emmons. The laminar-turbulent transition in a boundary layer-part i. Journal of the Aeronautical Sciences, 18(7):490-498, 1951.

[16] A. Favre. The equations of compressible turbulent gases. Technical Report AD0622097, DTIC Document, 1965.

[17] R. Fransen, E. Collado Morata, F. Duchaine, N. Gourdain, L. Gicquel, L. Vial, and G. Bonneau. Comparison of RANS and LES in high pressure turbines. In 3me Colloque INCA, ONERA, Toulouse, France, Nov, pages 17-18, 2011. 
[18] M. Gad-El-Hak, R. F. Blackwelderf, and J. J. Riley. On the growth of turbulent regions in laminar boundary layers. Journal of Fluid Mechanics, 110:73-95, 1981.

[19] A. Gehrer and Herbert H. Jericha. External heat transfer predictions in a highly loaded transonic linear turbine guide vane cascade using an upwind biased navier-stokes solver. Journal of Turbomachinery, 121(3):525-531, 1999.

[20] B. J. Geurts and J. Fröhlich. A framework for predicting accuracy limitations in large-eddy simulation. Physics of fluids, 14(6):L41-L44, 2002.

[21] N. Gourdain, L. Y. M. Gicquel, and E. Collado Morata. Comparison of RANS and LES for prediction of wall heat transfer in a highly loaded turbine guide vane. Journal of propulsion and power, 28(2): 423-433, 2012.

[22] V. Granet, O. Vermorel, T. Léonard, L. Gicquel, and T. Poinsot. Comparison of nonreflecting outlet boundary conditions for compressible solvers on unstructured grids. AIAA journal, 48(10):2348-2364, 2010.

[23] F. Guiho, F. Alizard, and J.-C. Robinet. Instabilities in oblique shock wave/laminar boundary-layer interactions. Journal of Fluid Mechanics, 789:1-35, 2016.

[24] R. J. Hakkinen, I. Greber, L. Trilling, and S. S. Abarbanel. The interaction of an oblique shock wave with a laminar boundary layer. NASA Memo 2-18-59W, 1356, 1959.

[25] N. Hildebrand, A. Dwivedi, J. W. Nichols, M. R. Jovanović, and G. V. Candler. Simulation and stability analysis of oblique shock-wave/boundary-layer interactions at mach 5.92. Physical Review Fluids, 3(1):013906, 2018.

[26] J.-C. Hoarau, P. Cinnella, and X. Gloerfelt. Large eddy simulation of dense gas flow around a turbine cascade. In AIAA Aviation 2019 Forum, page 2843, 2019.

[27] P. G. Huang, G. N. Coleman, and P. Bradshaw. Compressible turbulent channel flows: DNS results and modelling. Journal of Fluid Mechanics, 305:185-218, 1995.

[28] R. G. Jacobs and P. A. Durbin. Simulations of bypass transition. Journal of Fluid Mechanics, 428: $185-212,2001$.

[29] S. Jee, J. Joo, and G. Medic. Large-eddy simulation of a high-pressure turbine vane with inlet turbulence. In ASME Turbo Expo 2016: Turbomachinery Technical Conference and Exposition, pages V02DT44A019-V02DT44A019. American Society of Mechanical Engineers, 2016.

[30] L E. Jones, R. D. Sandberg, and N. D. Sandham. Stability and receptivity characteristics of a laminar separation bubble on an aerofoil. Journal of Fluid Mechanics, 648:257-296, 2010.

[31] Y. Katz, A. Seifert, and I. Wygnanski. On the evolution of the turbulent spot in a laminar boundary layer with a favourable pressure gradient. Journal of fluid mechanics, 221:1-22, 1990.

[32] R. H. Kraichnan. Diffusion by a random velocity field. The physics of fluids, 13(1):22-31, 1970.

[33] L. Krishnan and N. D. Sandham. Effect of mach number on the structure of turbulent spots. Journal of Fluid Mechanics, 566:225-234, 2006.

[34] L. Krishnan and N. D. Sandham. On the merging of turbulent spots in a supersonic boundary-layer flow. International Journal of Heat and Fluid Flow, 27(4):542-550, 2006.

[35] L. Krishnan and N. D. Sandham. Strong interaction of a turbulent spot with a shock-induced separation bubble. Physics of Fluids, 19(1):016102, 2007.

[36] G. M. Laskowski, J. Kopriva, V. Michelassi, S. Shankaran, U. Paliath, R. Bhaskaran, Q. Wang, C. Talnikar, Z. J. Wang, and F. Jia. Future directions of high fidelity CFD for aerothermal turbomachinery analysis and design. In 46th AIAA Fluid Dynamics Conference, page 3322, 2016.

[37] D. Lin, X. Yuan, and X. Su. Local entropy generation in compressible flow through a high pressure turbine with delayed detached eddy simulation. Entropy, 19(1):29, 2017.

[38] D. Lin, X. Su, and X. Yuan. The development and mechanisms of the high pressure turbine vane wake vortex. Journal of Engineering for Gas Turbines and Power, 140(9):092601, 2018.

[39] Y. Liu. Aerodynamics and heat transfer predictions in a highly loaded turbine blade. International Journal of Heat and Fluid Flow, 28(5):932-937, 2007.

[40] R. E. Mayle. The role of laminar-turbulent transition in gas turbine engines. Journal of Turbomachinery, 113(4):509-536, 1991.

[41] V. Michelassi, F. Martelli, R. Dénos, T. Arts, and C. H. Sieverding. Unsteady heat transfer in stator-rotor interaction by two equation turbulence model. In ASME 1998 International Gas Turbine and Aeroengine Congress and Exhibition, pages V001T01A058-V001T01A058. American Society of Mechanical Engineers, 1998.

[42] Z. Murphree, K. Yuceil, N. Clemens, and D. Dolling. Experimental studies of transitional boundary layer shock wave interactions. In 45th AIAA Aerospace Sciences Meeting and Exhibit, page 1139, 2006.

[43] R. Narasimha. The laminar-turbulent transition zone in the boundary layer. Progress in Aerospace Sciences, 22(1):29-80, 1985. 
[44] F. Nicoud and F. Ducros. Subgrid-scale stress modelling based on the square of the velocity gradient tensor. Flow, Turbulence and Combustion, 62(3):183-200, 1999. ISSN 1386-6184.

[45] K. P. Nolan and T. A. Zaki. Conditional sampling of transitional boundary layers in pressure gradients. Journal of Fluid Mechanics, 728:306-339, 2013.

[46] N. Odier, M. Sanjosé, L. Gicquel, T. Poinsot, S. Moreau, and F. Duchaine. A characteristic inlet boundary condition for compressible, turbulent, multispecies turbomachinery flows. Computers $\mathcal{G}$ Fluids, 178:41-55, 2019.

[47] A. Patel, B. J. Boersma, and R. Pecnik. Scalar statistics in variable property turbulent channel flows. Physical Review Fluids, 2(8):084604, 2017.

[48] R. Pecnik and A. Patel. Scaling and modelling of turbulence in variable property channel flows. Journal of Fluid Mechanics, 823, 2017.

[49] R. Pichler, J. Kopriva, G. Laskowski, V. Michelassi, and R. Sandberg. Highly resolved LES of a linear HPT vane cascade using structured and unstructured codes. In ASME Turbo Expo 2016: Turbomachinery Technical Conference and Exposition, pages V02CT39A041-V02CT39A041. American Society of Mechanical Engineers, 2016.

[50] R. Pichler, R. D. Sandberg, G. Laskowski, and V. Michelassi. High-fidelity simulations of a linear hpt vane cascade subject to varying inlet turbulence. In ASME Turbo Expo 2017: Turbomachinery Technical Conference and Exposition, pages V02AT40A001-V02AT40A001. American Society of Mechanical Engineers, 2017.

[51] S. B. Pope. Turbulent Flows. Cambridge University Press, 2000.

[52] T. J. Praisner and J. P. Clark. Predicting transition in turbomachinery-part i: A review and new model development. Journal of Turbomachinery, 129(1):1-13, 2007.

[53] T. J. Praisner, E. A. Grover, M. J. Rice, and J. P. Clark. Predicting transition in turbomachinery-Part II: Model validation and benchmarking. Journal of Turbomachinery, 129(1):14-22, 2007.

[54] R. Quadros and M. Bernardini. Numerical investigation of transitional shock-wave/boundary-layer interaction in supersonic regime. AIAA Journal, 56(7):2712-2724, 2018.

[55] M. Queguineur, L. Y. M. Gicquel, F. Dupuy, A. Misdariis, and G. Staffelbach. Dynamic mode tracking and control with a relaxation method. Physics of Fluids, 31(3):034101, 2019.

[56] J. A. Redford, N. D. Sandham, and G. T. Roberts. Numerical simulations of turbulent spots in supersonic boundary layers: effects of mach number and wall temperature. Progress in Aerospace Sciences, 52:67-79, 2012.

[57] B. Rehill, E. J. Walsh, L. Brandt, P. Schlatter, T. A. Zaki, and D. M. McEligot. Identifying turbulent spots in transitional boundary layers. Journal of turbomachinery, 135(1):011019, 2013.

[58] J. J. Riley and M. Gad-el-Hak. The dynamics of turbulent spots. In Frontiers in fluid mechanics, pages 123-155. Springer, 1985.

[59] J.-C. Robinet. Bifurcations in shock-wave/laminar-boundary-layer interaction: global instability approach. Journal of Fluid Mechanics, 579:85-112, 2007.

[60] R. D. Sandberg and V. Michelassi. The current state of high-fidelity simulations for main gas path turbomachinery components and their industrial impact. Flow, Turbulence and Combustion, 102(4): 797-848, 2019.

[61] R. D. Sandberg and A. P. S. Wheeler. Effect of trailing-edge boundary conditions on acoustic feedback loops in high-pressure turbines. Journal of Sound and Vibration, 461:114917, 2019.

[62] R. Sankaran and R. A. Antonia. Influence of a favorable pressure gradient on the growth of a turbulent spot. AIAA journal, 26(7):885-887, 1988.

[63] A. Sansica, N. D. Sandham, and Z. Hu. Instability and low-frequency unsteadiness in a shock-induced laminar separation bubble. Journal of Fluid Mechanics, 798:5-26, 2016.

[64] S. P. Schneider. Improved methods for measuring laminar-turbulent intermittency in boundary layers. Experiments in fluids, 18(5):370-375, 1995.

[65] T. Schönfeld and M. Rudgyard. Steady and unsteady flow simulations using the hybrid flow solver AVBP. AIAA journal, 37(11):1378-1385, 1999.

[66] G. B. Schubauer and P. S. Klebanoff. Contributions on the mechanics of boundarylayer transition. NACA Tech. Note, 3489, 1955.

[67] L. Segui, L. Gicquel, F. Duchaine, and J de Laborderie. LES of the LS89 cascade: influence of inflow turbulence on the flow predictions. In Proceedings of 12th European Conference on Turbomachinery Fluid dynamics and Thermodynamics ETC12. Stockholm, Sweden, pages 3-7, 2017.

[68] L. M. Segui, LYM Gicquel, F Duchaine, and J de Laborderie. Importance of boundary layer transition in a high-pressure turbine cascade using LES. In ASME Turbo Expo 2018: Turbomachinery Technical Conference and Exposition. American Society of Mechanical Engineers Digital Collection, 2018.

[69] A. Seifert and I. J. Wygnanski. On turbulent spots in a laminar boundary layer subjected to a self-similar adverse pressure gradient. Journal of Fluid Mechanics, 296:185-209, 1995. 
[70] R. L. Simpson. Turbulent boundary-layer separation. Annual Review of Fluid Mechanics, 21(1): 205-232, 1989.

[71] D. R. Stull and H. Prophet. Janaf thermochemical tables, 2nd edition. Technical Report NSRDS-NBS 37, US National Bureau of Standards, 1971.

[72] W. Sutherland. The viscosity of gases and molecular force. The London, Edinburgh, and Dublin Philosophical Magazine and Journal of Science, 36(223):507-531, 1893.

[73] K. Taira, S. L. Brunton, S. T. M. Dawson, C. W. Rowley, T. Colonius, B. J. McKeon, O. T. Schmidt, S. Gordeyev, V. Theofilis, and L. S. Ukeiley. Modal analysis of fluid flows: An overview. AIAA Journal, pages 4013-4041, 2017.

[74] J. Van den Eynde and J. Steelant. Compressibility and temperature effects on turbulent spot growth. Proceedings of HiSST, 2018.

[75] A. P. S. Wheeler, R. D. Sandberg, N. D. Sandham, R. Pichler, V. Michelassi, and G. Laskowski. Direct numerical simulations of a high-pressure turbine vane. Journal of Turbomachinery, 138(7): 071003, 2016.

[76] I. Wygnanski. The effects of Reynolds number and pressure gradient on the transitional spot in a laminar boundary layer. In The role of coherent structures in modelling turbulence and mixing, pages 304-332. Springer, 1981.

[77] B. Xiutao, W. Qingsong, S. Xinrong, and Y. Xin. Interaction mechanisms of shock waves with the boundary layer and wakes in a highly-loaded NGV using hybrid RANS/LES. Chinese Journal of Aeronautics, 2019.

[78] T. A. Zaki. From streaks to spots and on to turbulence: exploring the dynamics of boundary layer transition. Flow, turbulence and combustion, 91(3):451-473, 2013.

[79] M. Zauner, N. D. Sandham, A. Wheeler, and R. Sandberg. Linear stability prediction of vortex structures on high pressure turbine blades. International Journal of Turbomachinery, Propulsion and Power, 2(2):8, 2017.

[80] Y. Zhao and R. D. Sandberg. Bypass transition in boundary layers subject to strong pressure gradient and curvature effects. Journal of Fluid Mechanics, 888, 2020. 